\title{
A Vibration Reliability Analysis Method for the Uncertain Space Beam Structure
}

\author{
Yanyu Mo, ${ }^{1}$ Shuxiang Guo, ${ }^{2}$ and Cheng Tang ${ }^{2}$ \\ ${ }^{1}$ Aeronautics and Astronautics Engineering College, Air Force Engineering University, Xian 710038, China \\ ${ }^{2}$ Science College, Air Force Engineering University, Xian 710051, China
}

Correspondence should be addressed to Yanyu Mo; 14031875@qq.com

Received 1 September 2015; Revised 23 November 2015; Accepted 6 December 2015

Academic Editor: Miguel Neves

Copyright (C) 2016 Yanyu Mo et al. This is an open access article distributed under the Creative Commons Attribution License, which permits unrestricted use, distribution, and reproduction in any medium, provided the original work is properly cited.

Considering that uncertainty is inherent and unavoidable in engineering practice and the available information about the uncertain parameters is always not sufficient, the paper tries to carry out the nonprobabilistic vibration reliability analysis so as to avoid resonance on uncertain structure with bounded parameters. The input uncertain-but-bounded parameters are treated as interval variables, and an interval model is adopted to describe bounded uncertainties. Then a theory of nonprobabilistic reliability is introduced, in which the dimensionless nonprobabilistic reliability index and system reliability index are defined. In order to investigate the resonance failure with reliability method, the resonance failure domains are stated according to the relationships between the natural frequencies and the excitation frequencies. Then the uncertain structure is modeled as a series system and a system reliability index is proposed to evaluate the safety of the structure. The paper also takes a frequency analysis on the uncertain space beam structure to get the resonance failure modes. A frequency analysis method based on the monotonicity discriminant of the frequency sensitivity is presented. Then an optimization algorithm is introduced to verify the validity of the former frequency analysis method. Two examples are provided to illustrate the effectiveness and feasibility of the presented method.

\section{Introduction}

Uncertainty is inherent and unavoidable in almost all engineering problems. In structural engineering, uncertainties may present in structural materials, geometrical properties, theoretical modeling, failure conditions, applied loads, and so forth [1]. Reliability problems stem rightly from the existence of all these uncertainties. At present, in structural engineering, several mathematical theories are available for dealing with uncertainties, for example, probability theory, fuzzy set and evidence theory, interval analysis and convex-set models, and so forth. Then corresponding structural reliability method can be developed on the basis of these mathematical models. Considering that the method needs less sample data compared to the traditional probabilistic reliability method [2], the nonprobabilistic reliability method based on interval model has become one of the most widely used reliability methods in academic research and engineering in the past few years. Based on interval arithmetic, a nonprobabilistic measure and analysis methodology for structural reliability computation are presented by Shuxiang et al. [3]. Elishakoff and Ren [4] modeled structural uncertainties by both the stochastic and interval methods to quantify the uncertainties in response quantities. An interval stress and interval strength interference model for structural interval reliability analysis was investigated by Yang and Sun [5]. The investigation was based on a partition, which gave a full-scale description of comparison relations between interval strength and interval stress. A bilevel robust optimization model, combining interval extension of function algorithm and an order relation of interval number algorithm, was presented to investigate the effects of uncertainties on the objective and constraint functions for robust optimization by Sun et al. [6]. A new method was developed for reliability analysis of the uncertain structures with both random and interval variables by Jiang et al. [7]. And then a new nonprobabilistic structural reliability analysis method based on a multidimensional parallelepiped convex model was developed by Jiang et al. in the literature [8]. The model was constructed using marginal intervals for the variables and correlation information between any two 
variables. A nonprobabilistic robust reliability method for robust control of uncertain structures using static output feedback LQR approach was presented systematically by Guo and $\mathrm{Li}$ [9]. And a nonprobabilistic reliability methodology was presented systematically by Guo and Lu in the literature [10] for analysis and reliability-based design optimization of structures with uncertain-but-bounded parameters. Undoubtedly, the interval nonprobabilistic reliability idea based on set models is creative and significant. Recently, some scholars utilize reliability method to avoid resonance of the structure. Zhang et al. [11] presented the probability of the resonance state of the structure which was regarded as a series system. Su et al. [12] carried out a vibration reliability analysis method for avoiding the resonance and studied the effects of random parameters on vibration reliability of the resonance structure. The reliability methods employed in the literature $[11,12]$ are both traditional probabilistic reliability methods. Considering that the available information about the uncertain parameters may be not sufficient in engineering practice, the interval nonprobabilistic reliability method should be a good choice for resonance avoiding.

In order to define the resonance failure modes of the uncertain structure, a frequency analysis which aims to get the upper and lower bounds of the natural frequencies is needed. It is also known as the interval eigenvalue problem which has aroused the interest of many scholars. Rohn [13] studied the generalized interval eigenvalue problem and derived formulas for the interval eigenvalue of a symmetric interval matrix with an error matrix of rank one. Based on the invariance properties of the characteristic vector entries, Deif [14] presented a method to compute interval eigenvalues for the standard interval eigenvalue problem. Qiu et al. [15] extended Deif's method to the generalized interval eigenvalue problem. By viewing the deviation amplitudes of the mass and stiffness matrices as perturbations around the nominal values of the interval matrix pair, a perturbation method for the solution of the generalized interval eigenvalues problem has been developed by Qiu et al. [16]. Elishakoff [17] proposed a procedure for finding the range of eigenvalues due to uncertain elastic modulus and mass density by using the upper and lower stiffness and mass matrices. Gao [18] proposed the interval factor method to investigate the effects of geometrical and material interval uncertainties on the natural frequencies and mode shapes of truss structures. Modares et al. [19] proved that, in the presence of any physically allowable uncertainty in the structural stiffness, the solutions of two deterministic eigenvalue problems are sufficient to obtain the exact bounds of the system's fundamental frequencies without resort to any combinatorial solution procedures. Sofi et al. [20] presented an efficient procedure for the solution of the generalized interval eigenvalue problem arising from vibration analysis of linear undamped structures with uncertain-but-bounded parameters.

The aim of this paper is to propose an efficient method for the frequency analysis of the space beam structure and take an interval nonprobabilistic reliability analysis of avoiding resonance on the uncertain structure. The remainder of this paper is organized as follows. In Section 2, a series system vibration reliability based on the nonprobabilistic reliability, different from the traditional probabilistic reliability, is introduced. An analysis on the failure modes of resonance in uncertain structure is carried out in Section 3, and the system reliability index of the resonance structure is obtained. In Section 4, a frequency analysis on the space beam structure is carried out and an efficient frequency analysis method is presented. A space frame structure and a simplified GARTEUR plane model are used to demonstrate the presented methods in Section 5, and finally conclusions are given in Section 6.

\section{Overview of the Nonprobabilistic Reliability Method Based on Interval Model}

Interval model is one of the most frequently used models in uncertainty analysis of structures. The model describes the uncertain-but-bounded parameters as interval variables $\mathbf{X}=$ $\left(X_{1}, X_{2}, \ldots, X_{n}\right)$ satisfying $X_{i} \in\left[X_{i}^{L}, X_{i}^{U}\right](i=1,2, \ldots, n)$, where $X_{i}^{L}$ is the lower bound of $X_{i}$ and $X_{i}^{U}$ is the upper bound of it. Then the interval variables can be transformed into standard ones by the following normalized transformation:

$$
X_{i}=X_{i c}+\delta_{X i} \cdot X_{i r}, \quad i=1,2, \ldots, n,
$$

where

$$
\begin{aligned}
& X_{i c}=\frac{\left(X_{i}^{U}+X_{i}^{L}\right)}{2}, \\
& X_{i r}=\frac{\left(X_{i}^{U}-X_{i}^{L}\right)}{2}, \\
& \quad i=1,2, \ldots, n,
\end{aligned}
$$

and $\delta_{X i} \in[-1,1]$ denotes the standard interval variables corresponding to $X_{i} . X_{i c}$ is the mean value of $X_{i}$, and $X_{i c}$ is the deviation of it.

2.1. Measure of Nonprobabilistic Reliability. In structural reliability analysis, a limit-state function (LSF) $Z=g(\mathbf{X})$ is usually defined by the performance or failure mode of the structure. $g(\mathbf{X})$ is usually a function of the input variables $\mathbf{X}=\left(X_{1}, X_{2}, \ldots, X_{n}\right)$, with $g(\mathbf{X})=0$ being the limit-state surface (LSS) which separates the variable space into two domains: the failure domain $\Omega_{f}=\{\mathbf{X} \mid g(\mathbf{X})<0\}$ and the safe domain $\Omega_{s}=\{\mathbf{X} \mid g(\mathbf{X})>0\}$.

In the situations that all the variables $\mathbf{X}=\left(X_{1}, X_{2}\right.$, $\left.\ldots, X_{n}\right)$ involved in the reliability problem under consideration can be bounded in certain intervals and represented by interval variables, as done above, all the interval variables are transformed into the standard interval variables $\boldsymbol{\delta}_{\mathbf{X}}=$ $\left(\delta_{\mathbf{X} 1}, \delta_{\mathbf{X} 2}, \ldots, \delta_{\mathbf{X}_{n}}\right)$ by means of transformation in (1). Then the LSF $Z=g(\mathbf{X})$ can be transformed into the following normalized form [10]:

$$
Z=g(\mathbf{X})=G\left(\boldsymbol{\delta}_{\mathbf{X}}\right),
$$

and the reliability index can be defined as follows [3]:

$$
\eta=\min \left\{\left\|\boldsymbol{\delta}_{\mathbf{X}}\right\|_{\infty} \mid G\left(\boldsymbol{\delta}_{\mathbf{X}}\right)=0\right\},
$$

where $\|\cdot\|_{\infty}$ denotes the infinity norm of vector. 


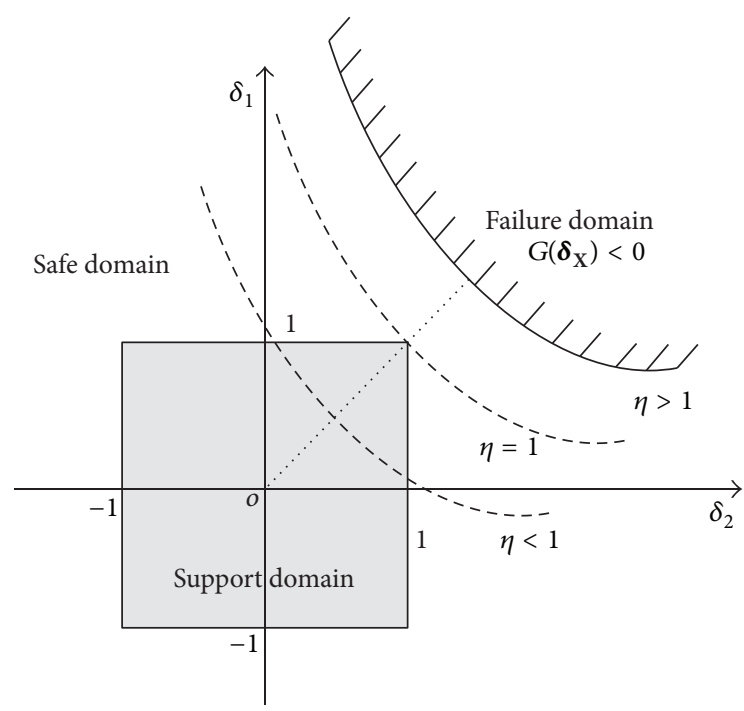

FIGURE 1: The nonprobabilistic reliability based on two-dimensional interval model.

It can be seen that the nonprobabilistic reliability index $\eta$ in (4) represents the minimum distance which is measured by the infinity norm from the origin to the LSS (or to the failure domain) in the infinite topological space built by all the standard interval variables. The reliability index $\eta$ is illustrated schematically by the two-dimensional case in Figure 1. When $\eta>1$, the structure keeps safety; when $\eta=1$, the structure is in the critical state; and when $0<\eta<1$, part of the support domain is in the failure domain, the structure is considered to be unsafe for that it can't complete expected function convincingly. The case when $\eta \leq 0$ is not included in the definition; it will be meaningless from the point of view of the reliability.

2.2. Nonprobabilistic Reliability Index of a Structure System. In reliability analysis of a structure system, multiple LSFs which are defined by failure modes of the structure are usually involved. Assuming that there exist $m$ potential significant failure modes which need to be taken into account, the corresponding LSFs are represented by $Z_{k}=g_{k}(\mathbf{X})(k=$ $1,2, \ldots, m)$, where $Z_{k}<0$ indicates the $k$ th failure mode. The structural system is usually regarded as a series system consisting of significant failure modes, because the occurrence of any one of the failure modes may lead to the failure of the structural system. Suppose that the input variables $\mathbf{X}=\left(X_{1}, X_{2}, \ldots, X_{n}\right)$ are independent interval variables. Transform all the interval variables into the standard ones $\boldsymbol{\delta}_{\mathrm{X}}=\left(\delta_{\mathrm{X} 1}, \delta_{\mathrm{X} 2}, \ldots, \delta_{\mathrm{X}_{n}}\right)$ by means of (1) and then substitute that into the LSFs. Then the standardized LSFs $Z_{k}=G_{k}\left(\boldsymbol{\delta}_{\mathrm{X}}\right)$ $(k=1,2, \ldots, m)$ can be obtained. The nonprobabilistic reliability index of the structural system can be given as follows [3]:

$$
\eta_{\text {sys }}=\min \left\{\eta_{k}\right\}, \quad k=1,2, \ldots, m,
$$

where $\eta_{k}$ is the nonprobabilistic reliability index corresponding to the $k$ th LSF. The reliability index $\eta_{\text {sys }}$ of the structure

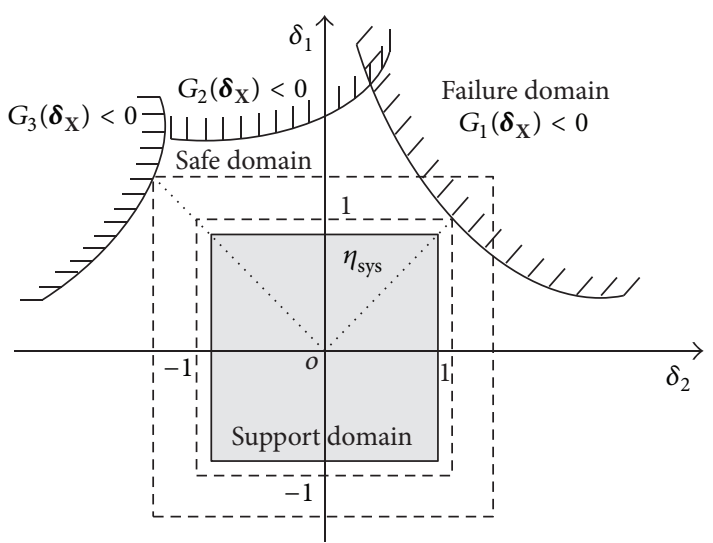

FIGURE 2: The nonprobabilistic reliability of a structure system.

system is illustrated schematically by the two-dimensional case in Figure 2. It can be seen from Figure 2 that $\eta_{\text {sys }}$ is the shortest distance from the origin to the failure surface. $\eta_{\text {sys }}$ corresponds to the most likely occurred failure mode. So it is reasonable to take $\eta_{\text {sys }}$ in (5) as the measure of the structure system.

\section{Nonprobabilistic Reliability of the Uncertain Resonance Structure}

As we all know, resonance occurs when excitation frequency is close to the natural frequency of a vibrating system. The significant phenomenon of resonance is that the vibration amplitude and energy sharply increase, which may lead to the failure of the structure. The failure is called resonance failure here.

3.1. State Function of the Failure Resonance. Resonance failure is different from the first passage problems [21]; it is also an important failure problem in vibration analysis. When resonance occurs, the vibration amplitude increases obviously, especially when the damp of the structure is low. The failure of resonance has two cases; one of them is that the amplitude is beyond the threshold, and the other is that the amplitude does not exceed the threshold due to the existence of damp [11]. In the first case, the structure is surely failure; in the second case, the propagation of fatigue crack is much accelerated by the resonance [22]; the structure is also regarded as failure here.

According to the reliability theory, the state function of failure resonance can be expressed as

$$
g_{i, j}=\left|P_{i}-\omega_{j}\right|-\gamma, \quad i=1,2, \ldots, m ; j=1,2, \ldots, n,
$$

where $P_{i}$ denotes the frequency of the $i$ th excitation, $\omega_{j}$ denotes the $j$ th natural frequency of the structure, and $\gamma$ represents the critical spacing between natural frequency and excitation frequency. When the spacing between natural frequency and excitation frequency is less than $\gamma$, the resonance occurs. To the authors' knowledge, the value of $\gamma$ is related to the damp of the structure. The value of $\gamma$ should be reasonably 


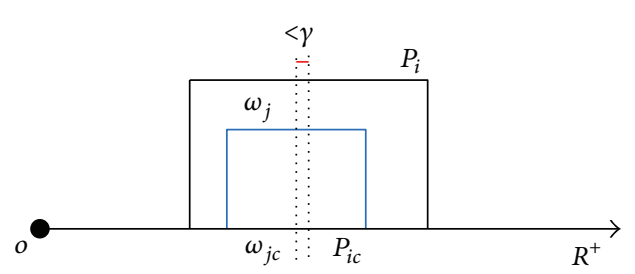

(a)

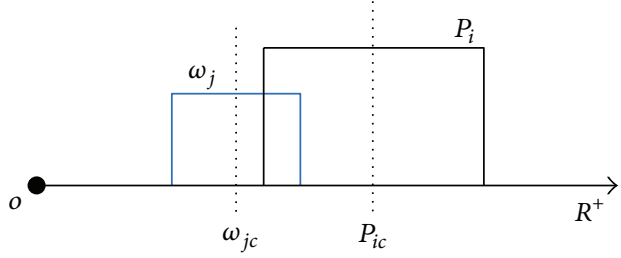

(c)

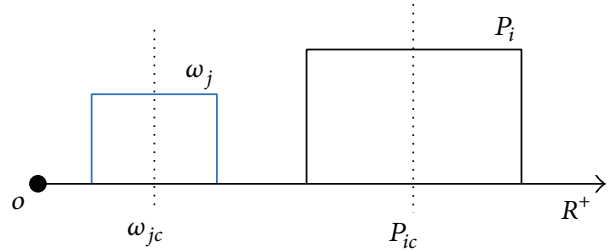

(e)

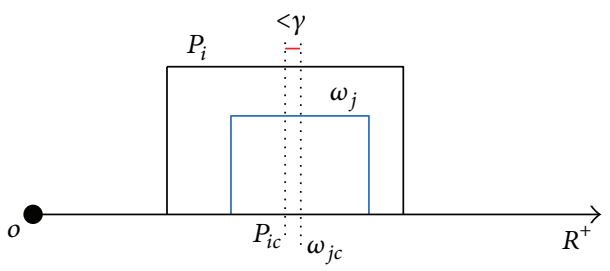

(b)

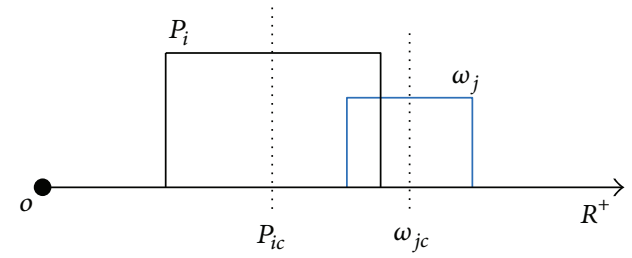

(d)

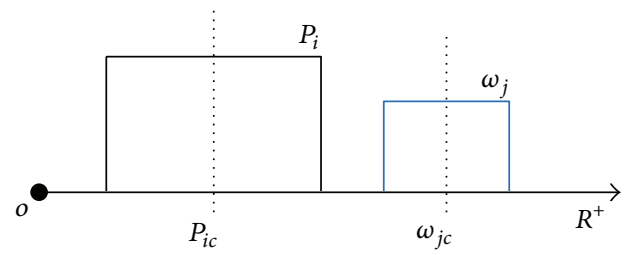

(f)

FIgure 3: Relationships between $P_{i}$ and $\omega_{j}$. (a) $0 \leq P_{i c}-\omega_{j c} \leq \gamma$, overlapping. (b) $-\gamma \leq P_{i c}-\omega_{j c} \leq 0$, overlapping. (c) $P_{i c}>\omega_{j c}$, overlapping. (d) $P_{i c}<\omega_{j c}$, overlapping. (e) $P_{i c}>\omega_{j c}$, not overlapping. (f) $P_{i c}<\omega_{j c}$, not overlapping.

defined in accordance with the effect of the amplitude on the structure and may be obtained based on previous experience in engineering practice. In this paper, a hypothetical value $1.5 \mathrm{~Hz}$ is given to $\gamma$.

3.2. Failure Mode of Resonance in Uncertain Structure. For uncertain structures with interval parameters, the natural frequencies are implicit functions of the interval variables. So the natural frequencies $\omega_{j}$ surely are interval numbers. Assuming that the excitation frequencies $P_{i}$ are also interval numbers, the failure mode can be expressed as follows according to the state function in (6):

$$
g_{i, j}=\left|P_{i}-\omega_{j}\right|-\gamma<0,
$$

where $P_{i}$ and $\omega_{j}$ can be transformed into standard ones as follows by means of (1):

$$
\begin{gathered}
P_{i}=P_{i c}+\delta_{P i} \cdot P_{i r}, \quad i=1,2, \ldots, m ; \\
\omega_{j}=\omega_{j c}+\delta_{\omega j} \cdot \omega_{j r}, \quad j=1,2, \ldots, n .
\end{gathered}
$$

$P_{i c}$ and $\omega_{j c}$ denote the mean value of $P_{i}$ and $\omega_{j} ; P_{i r}$ and $\omega_{j r}$ denote the deviation of $P_{i}$ and $\omega_{j}$, respectively; $\delta_{P i}$ and $\delta_{\omega j}$ are standard interval number equal to $[-1,1]$. Substituting $(8)$ into (7), the expression of failure mode can be expressed as

$$
\begin{aligned}
G_{i, j}=\left|P_{i c}+\delta_{P i} \cdot P_{i r}-\omega_{j c}-\delta_{\omega j} \cdot \omega_{j r}\right|-\gamma<0, & \\
i=1,2, \ldots, m ; j & =1,2, \ldots, n,
\end{aligned}
$$

where $G_{i, j}=0$ denotes the two LSSs.
The failure modes are different due to the different relationships between $P_{i}$ and $\omega_{j}$. Six different relationships are illustrated schematically in Figure 3.

The failure modes corresponding to different cases in Figure 3 are illustrated in Figure 4.

From Figure 4, it can be seen that the failure domains of resonance are infinite strip areas. In Figures 4(a) and 4(b), the origin of the standard space is in the failure domain. The structure in these two cases is considered to be failure for the absolute value of the difference between the mean value $P_{i c}$ and $\omega_{j c}$ is less than the critical spacing $\gamma$ and the reliability index $\eta_{i, j}$ is zero. In Figures 4(c) and 4(d), the failure domain passes through the support domain on either side of the origin, and the reliability index $\eta_{i, j}$ can be measured by the minimum distance (measured by the infinity norm) from the origin to the failure domain. In Figures 4(e) and 4(f), the midcourt line of the failure domain is out of the support domain, and the reliability index $\eta_{i, j}$ can also be measured by the minimum distance from the origin to the failure domain. From Figure 4, it can also be known that the reliability index $\eta_{i, j}$ is the distance from the origin to upper boundary of the failure domain when the mean value $P_{i c}>\omega_{j c}$, or the distance from the origin to lower boundary of the failure domain when the mean value $P_{i c}<\omega_{j c}$. The reliability index $\eta_{i, j}$ can be expressed as follows according to (4):

$$
\begin{aligned}
\eta_{i, j}=\min \left\{\|\boldsymbol{\delta}\|_{\infty} \mid G_{i, j}\right. & \left.=0, \boldsymbol{\delta}=\left(\delta_{P i}, \delta_{\omega j}\right)\right\}, \\
& i=1,2, \ldots, m ; j=1,2, \ldots, n,
\end{aligned}
$$




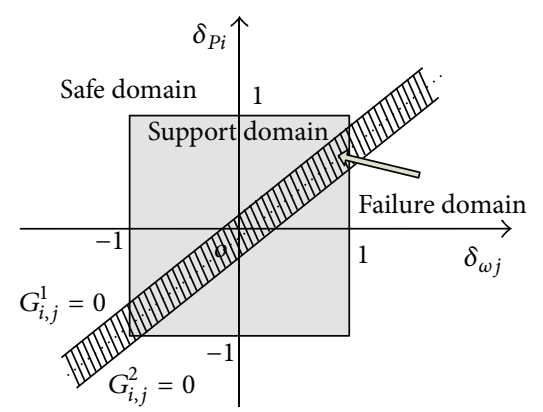

(a)

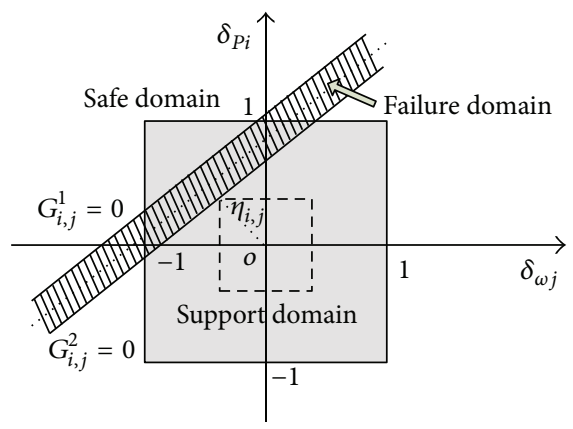

(d)

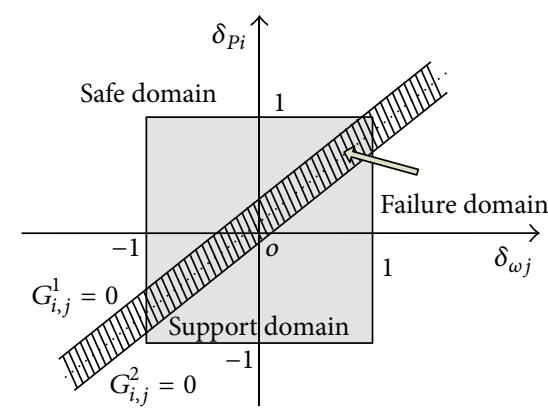

(b)

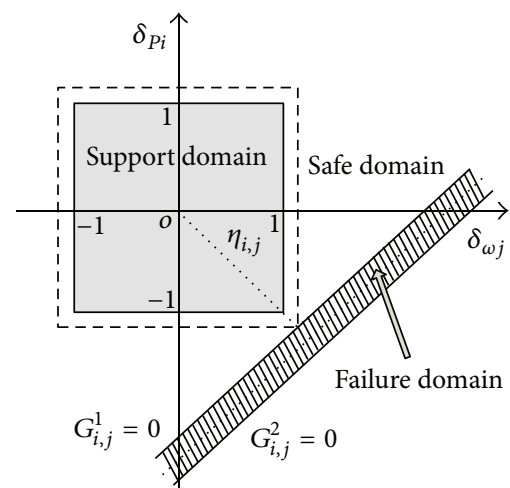

(e)

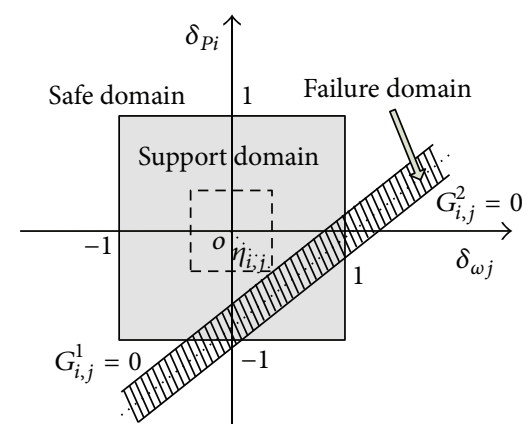

(c)

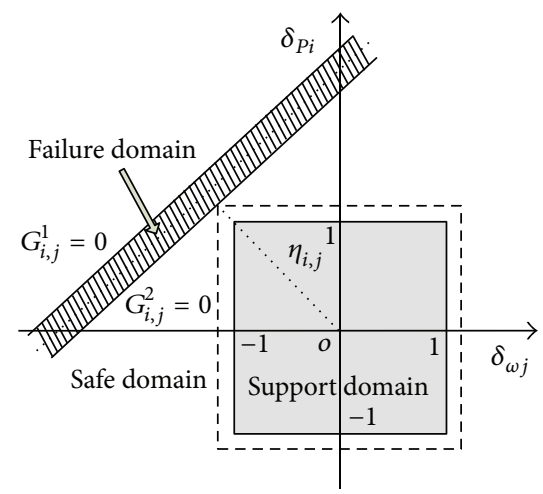

(f)

FIGURE 4: The failure modes of resonance corresponding to different relationships between $P_{i}$ and $\omega_{j}$. (a) $0 \leq P_{i c}-\omega_{j c} \leq \gamma$, overlapping. (b) $-\gamma \leq P_{i c}-\omega_{j c} \leq 0$, overlapping. (c) $P_{i c}>\omega_{j c}$, overlapping. (d) $P_{i c}<\omega_{j c}$, overlapping. (e) $P_{i c}>\omega_{j c}$, not overlapping. (f) $P_{i c}<\omega_{j c}$, not overlapping.

where $\boldsymbol{\delta}$ denotes the vector $\left(\delta_{P i}, \delta_{\omega j}\right)$ and $G_{i, j}=0$ denote the LSSs.

\subsection{Nonprobabilistic Reliability of the Uncertain Structure with} Resonance Failure. Assuming that the uncertain structure has $m$ natural frequencies, with $n$ excitation frequencies loading on it, the whole structure is considered to be in failure states because the resonance might occur when the excitation frequency is in the vicinity of any one of the natural frequencies. Thus, the structure system, to which excitation frequencies and natural frequencies are applied to analyze failure state, is a series system [11]. The system reliability index is presented as follows according to (5):

$$
\eta_{\text {sys }}=\min \left\{\eta_{i, j}\right\}, \quad i=1,2, \ldots, m ; j=1,2, \ldots, n .
$$

System reliability index $\eta_{\text {sys }}$ is the minimum of $\eta_{i, j}$, and it represents the most dangerous case for the structure when resonance occurs.

\section{Frequency Analysis of Uncertain Space Beam Structure}

4.1. The Rayleigh Quotient Based on Finite Element Analysis. For a space beam structure with $s$ degrees of freedom, the natural frequency can be obtained by solving the following generalized Rayleigh quotient [23]:

$$
\lambda_{t}=\frac{\boldsymbol{\varphi}_{t}^{T} \mathbf{K} \boldsymbol{\varphi}_{t}}{\boldsymbol{\varphi}_{t}^{T} \mathbf{M} \boldsymbol{\varphi}_{t}}, \quad t=1,2, \ldots, s,
$$

where $\lambda_{t}$ denotes the square of the $t$ th order natural frequency; $s$ denotes the number of degrees of freedom; $\mathbf{K}$ denotes the global stiffness matrix of the structure, which is a $s$ order real symmetric positive semidefinite matrix; $\mathbf{M}$ denotes the global mass matrix of the structure, which is a $s$ order real symmetric positive definite matrix; $\boldsymbol{\varphi}_{t}$ denotes the dimensionless $t$ th order standard mode.

Taking Euler-Bernoulli beam structure into consideration, $n$ space beam elements with $m$ nodes are obtained by carrying a finite element analysis on the structure. And the global stiffness matrix and global mass matrix of the structure can be expressed as follows:

$$
\begin{aligned}
\mathbf{K}= & \sum_{i=1}^{n} \mathbf{K}_{i}^{\mathrm{ele}} \\
= & \sum_{i=1}^{n} E_{i} A_{i} \mathbf{K}_{i(1)}^{\mathrm{ele}}+\sum_{i=1}^{n} E_{i} I_{z i} \mathbf{K}_{i(2)}^{\mathrm{ele}}+\sum_{i=1}^{n} E_{i} I_{y i} \mathbf{K}_{i(3)}^{\mathrm{ele}} \\
& +\sum_{i=1}^{n} G_{i} J_{i} \mathbf{K}_{i(4)}^{\mathrm{ele}},
\end{aligned}
$$




$$
\begin{aligned}
\mathbf{M} & =\sum_{i=1}^{n} \mathbf{M}_{i}^{\mathrm{ele}} \\
& =\sum_{i=1}^{n} \rho_{i} A_{i}\left(\mathbf{M}_{i(1)}^{\mathrm{ele}}+\mathbf{M}_{i(2)}^{\mathrm{ele}}+\mathbf{M}_{i(3)}^{\mathrm{ele}}\right)+\sum_{i=1}^{n} \rho_{i} J_{i} \mathbf{M}_{i(4)}^{\mathrm{ele}},
\end{aligned}
$$

where $\mathbf{K}$ denotes the $6 m \times 6 m$ global stiffness matrix; $\mathbf{M}$ denotes the $6 m \times 6 m$ global mass matrix; $A_{i}$ is the beam crosssection area of the $i$ th element; $I_{z i}$ is the $z$ axial moment of inertia; $I_{y i}$ is the $y$ axial moment of inertia; $J_{i}$ is the polar moment of inertia; and $L_{i}$ is the beam element length. For isotropic material, $G_{i}=E_{i} /\left(2\left(1+\mu_{i}\right)\right)$ holds. $E_{i}$ is the elasticity modulus of the $i$ th element; $\mu_{i}$ is Poisson's ratio and $\rho_{i}$ is the density; $\mathbf{K}_{i}^{\text {ele }}$ is the expanded $6 m \times 6 m$ element stiffness matrix of the $i$ th element; $\mathbf{M}_{i}^{\text {ele }}$ is the expanded $6 m \times 6 m$ element mass matrix of the $i$ th element; $\mathbf{K}_{i(1)}^{\text {ele }}, \mathbf{K}_{i(2)}^{\text {ele }}, \mathbf{K}_{i(3)}^{\text {ele }}$, and $\mathbf{K}_{i(4)}^{\text {ele }}$ denote the component matrices of $\mathbf{K}_{i}^{\mathrm{ele}}$, and $\mathbf{M}_{i(1)}^{\mathrm{ele}}, \mathbf{M}_{i(2)}^{\mathrm{ele}}, \mathbf{M}_{i(3)}^{\mathrm{ele}}$, and $\mathbf{M}_{i(4)}^{\text {ele }}$ denote the component matrices of $\mathbf{M}_{i}^{\text {ele }}$. The superscript "ele" stands for the beam element with respect to any matrix following it.

The matrices $\mathbf{K}_{i(1)}^{\text {ele }}, \mathbf{K}_{i(2)}^{\mathrm{ele}}, \mathbf{K}_{i(3)}^{\mathrm{ele}}, \mathbf{K}_{i(4)}^{\mathrm{ele}}, \mathbf{M}_{i(1)}^{\mathrm{ele}}, \mathbf{M}_{i(2)}^{\mathrm{ele}}, \mathbf{M}_{i(3)}^{\mathrm{ele}}$, and $\mathbf{M}_{i(4)}^{\text {ele }}$ are given as follows in terms of finite element method:

$$
\begin{aligned}
& \mathbf{K}_{i(1)}^{\text {ele }}=\frac{1}{L_{i}} *\left[\begin{array}{ccccccc}
0 & \cdots & 0 & \cdots & 0 & \cdots & 0 \\
\vdots & & \vdots & & \vdots & & \vdots \\
0 & \cdots & 1 & \cdots & -1 & \cdots & 0 \\
\vdots & & \vdots & & \vdots & & \vdots \\
0 & \cdots & -1 & \cdots & 1 & \cdots & 0 \\
\vdots & & \vdots & & \vdots & & \vdots \\
0 & \cdots & 0 & \cdots & 0 & \cdots & 0
\end{array}\right]_{6 m \times 6 m} \\
& \mathbf{K}_{i(2)}^{\mathrm{ele}}=\left[\begin{array}{ccccccccc}
0 & & \cdots & & \cdots & & \cdots & & 0 \\
& \frac{12}{L_{i}^{3}} & \cdots & \frac{6}{L_{i}^{2}} & \cdots & -\frac{12}{L_{i}^{3}} & \cdots & \frac{6}{L_{i}^{2}} & \\
\vdots & \vdots & & \vdots & & \vdots & & \vdots & \vdots \\
& \frac{6}{L_{i}^{2}} & \cdots & \frac{4}{L_{i}} & \cdots & -\frac{6}{L_{i}^{2}} & \cdots & \frac{2}{L_{i}} & \\
\vdots & \vdots & & \vdots & & \vdots & & \vdots & \vdots \\
& -\frac{12}{L_{i}^{3}} & \cdots & -\frac{6}{L_{i}^{2}} & \cdots & \frac{12}{L_{i}^{3}} & \cdots & -\frac{6}{L_{i}^{2}} & \\
\vdots & \vdots & & \vdots & & \vdots & & \vdots & \vdots \\
& \frac{6}{L_{i}^{2}} & \cdots & \frac{2}{L_{i}} & \cdots & -\frac{6}{L_{i}^{2}} & \cdots & \frac{4}{L_{i}} & \\
0 & & \cdots & & \cdots & & \cdots & & 0
\end{array}\right]_{6 m \times 6 m} \\
& \mathbf{K}_{i(4)}^{\text {ele }}=\frac{1}{L_{i}} *\left[\begin{array}{ccccccc}
0 & \cdots & 0 & \cdots & 0 & \cdots & 0 \\
\vdots & & \vdots & & \vdots & & \vdots \\
0 & \cdots & 1 & \cdots & -1 & \cdots & 0 \\
\vdots & & \vdots & & \vdots & & \vdots \\
0 & \cdots & -1 & \cdots & 1 & \cdots & 0 \\
\vdots & & \vdots & & \vdots & & \vdots \\
0 & \cdots & 0 & \cdots & 0 & \cdots & 0
\end{array}\right]_{6 m \times 6 m}
\end{aligned}
$$




$$
\begin{aligned}
& \mathbf{K}_{i(3)}^{\mathrm{ele}}=\left[\begin{array}{ccccccccc}
0 & & \cdots & & \cdots & & \cdots & & 0 \\
& \frac{12}{L_{i}^{3}} & \cdots & -\frac{6}{L_{i}^{2}} & \cdots & -\frac{12}{L_{i}^{3}} & \cdots & -\frac{6}{L_{i}^{2}} & \\
\vdots & \vdots & & \vdots & & \vdots & & \vdots & \vdots \\
& -\frac{6}{L_{i}^{2}} & \cdots & \frac{4}{L_{i}} & \cdots & \frac{6}{L_{i}^{2}} & \cdots & \frac{2}{L_{i}} & \\
\vdots & \vdots & & \vdots & & \vdots & & \vdots & \vdots \\
& -\frac{12}{L_{i}^{3}} & \cdots & \frac{6}{L_{i}^{2}} & \cdots & \frac{12}{L_{i}^{3}} & \cdots & \frac{6}{L_{i}^{2}} & \\
\vdots & \vdots & & \vdots & & \vdots & & \vdots & \vdots \\
& -\frac{6}{L_{i}^{2}} & \cdots & \frac{2}{L_{i}} & \cdots & \frac{6}{L_{i}^{2}} & \cdots & \frac{4}{L_{i}} & \\
0 & & \cdots & & \cdots & & \cdots & & 0
\end{array}\right]_{6 m \times 6 m}
\end{aligned}
$$

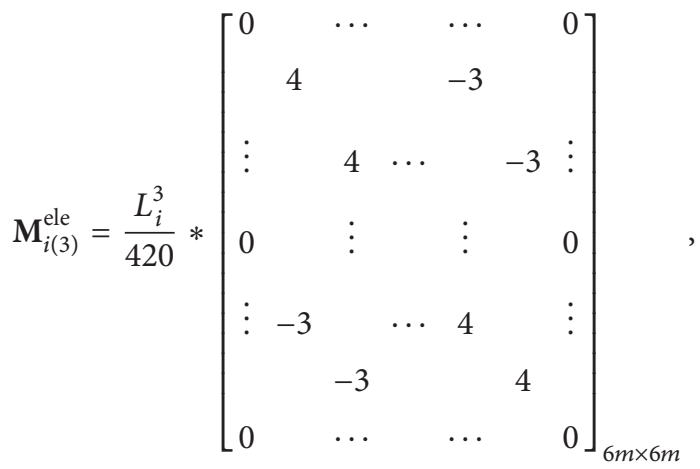

$$
\begin{aligned}
& \mathbf{M}_{i(1)}^{\text {ele }}=\frac{L_{i}}{420} *\left[\begin{array}{ccccccccc}
0 & & \cdots & & \cdots & & \cdots & & 0 \\
& & & & & & & \vdots & \\
& 140 & & \cdots & & 70 & & \vdots & \\
\vdots & & 156 & & & 54 & & \vdots \\
& \vdots & & 156 & & \vdots & & 54 & \\
\vdots & & \vdots & & & & \vdots & & \vdots \\
& & & \vdots & & & & & \\
\vdots & & 54 & & & & 156 & & \vdots \\
& \vdots & & 54 & & \ldots & & 156 & \\
0 & & \cdots & & \cdots & & \cdots & & 0
\end{array}\right]_{6 m \times 6 m} \\
& \mathbf{M}_{i(4)}^{\text {ele }}=\frac{L_{i}}{420} *\left[\begin{array}{ccccccc}
0 & \cdots & 0 & \cdots & 0 & \cdots & 0 \\
\vdots & & \vdots & & \vdots & & \vdots \\
0 & \cdots & 140 & \cdots & 70 & \cdots & 0 \\
\vdots & & \vdots & & \vdots & & \vdots \\
0 & \cdots & 70 & \cdots & 140 & \cdots & 0 \\
\vdots & & \vdots & & \vdots & & \vdots \\
0 & \cdots & 0 & \cdots & 0 & \cdots & 0
\end{array}\right]_{6 m \times 6 m}, \text {, }
\end{aligned}
$$




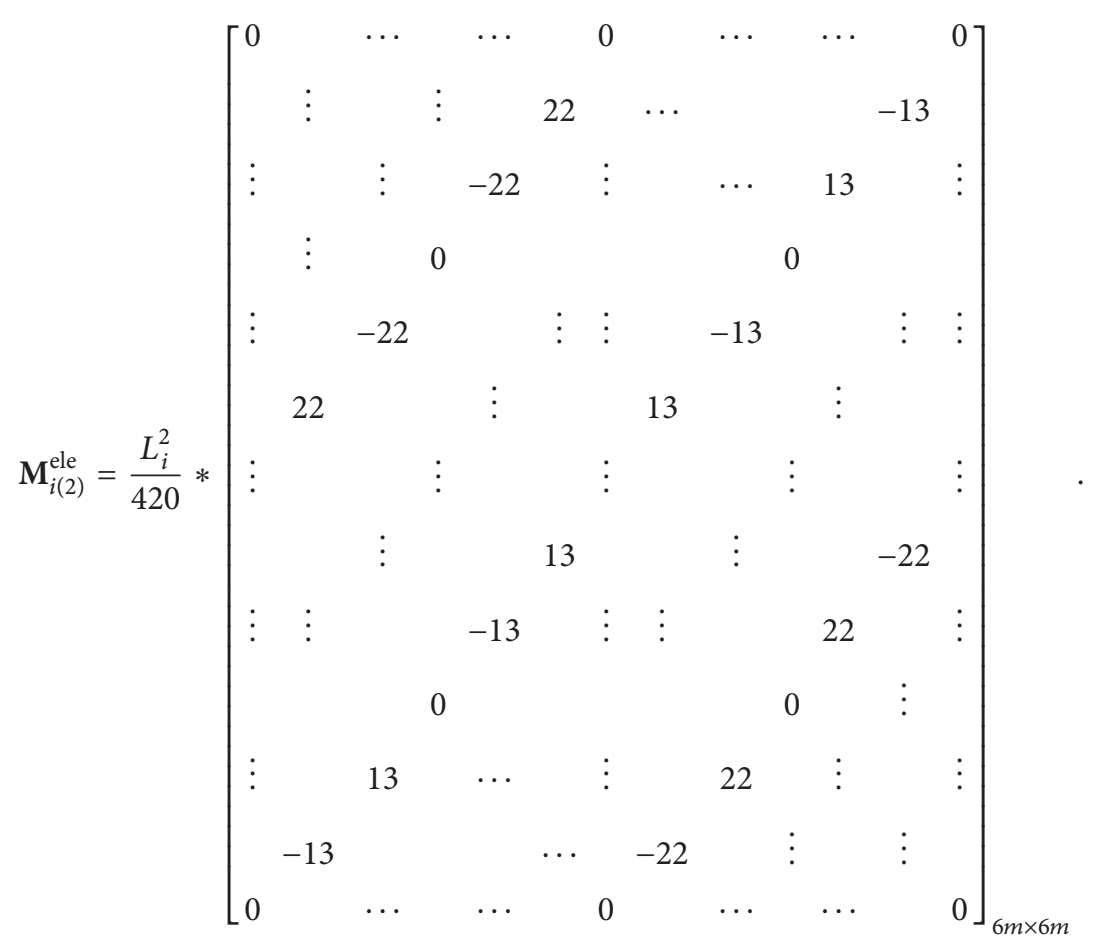

Substituting $\mathbf{K}$ and $\mathbf{M}$ in (13) into (12), the square of the $t$ th order natural frequency is deduced as follows:

$$
\begin{aligned}
\lambda_{t}= & \frac{\boldsymbol{\varphi}_{t}^{T}\left(\sum_{i=1}^{n} E_{i} A_{i} \mathbf{K}_{i(1)}^{\mathrm{ele}}\right) \boldsymbol{\varphi}_{t}}{\boldsymbol{\varphi}_{t}^{T}\left(\sum_{i=1}^{n} \mathbf{M}_{i}^{\mathrm{ele}}\right) \boldsymbol{\varphi}_{t}} \\
& +\frac{\boldsymbol{\varphi}_{t}^{T}\left(\sum_{i=1}^{n} E_{i} I_{z i} \mathbf{K}_{i(2)}^{\mathrm{ele}}\right) \boldsymbol{\varphi}_{t}}{\boldsymbol{\varphi}_{t}^{T}\left(\sum_{i=1}^{n} \mathbf{M}_{i}^{\mathrm{ele}}\right) \boldsymbol{\varphi}_{t}} \\
& +\frac{\boldsymbol{\varphi}_{t}^{T}\left(\sum_{i=1}^{n} E_{i} I_{y i} \mathbf{K}_{i(3)}^{\mathrm{ele}}\right) \boldsymbol{\varphi}_{t}}{\boldsymbol{\varphi}_{t}^{T}\left(\sum_{i=1}^{n} \mathbf{M}_{i}^{\mathrm{ele}}\right) \boldsymbol{\varphi}_{t}} \\
& +\frac{\boldsymbol{\varphi}_{t}^{T}\left(\sum_{i=1}^{n} G_{i} J_{i} \mathbf{K}_{i(4)}^{\mathrm{ele}}\right) \boldsymbol{\varphi}_{t}}{\boldsymbol{\varphi}_{t}^{T}\left(\sum_{i=1}^{n} \mathbf{M}_{i}^{\mathrm{ele}}\right) \boldsymbol{\varphi}_{t}} \\
= & \sum_{i=1}^{n} E_{i} \cdot \lambda_{i(1), t}+\sum_{i=1}^{n} E_{i} \cdot \lambda_{i(2), t}+\sum_{i=1}^{n} E_{i} \cdot \lambda_{i(3), t} \\
& +\sum_{i=1}^{n} \frac{E_{i}}{2\left(1+\mu_{i}\right)} \cdot \lambda_{i(4), t},
\end{aligned}
$$

where

$$
\begin{aligned}
& \lambda_{i(1), t}=\frac{\boldsymbol{\varphi}_{t}^{T}\left(A_{i} \mathbf{K}_{i(1)}^{\mathrm{ele}}\right) \boldsymbol{\varphi}_{t}}{\boldsymbol{\varphi}_{t}^{T}\left(\sum_{i=1}^{n} \mathbf{M}_{i}^{\mathrm{ele}}\right) \boldsymbol{\varphi}_{t}}, \\
& \lambda_{i(2), t}=\frac{\boldsymbol{\varphi}_{t}^{T}\left(I_{z i} \mathbf{K}_{i(2)}^{\mathrm{ele}}\right) \boldsymbol{\varphi}_{t}}{\boldsymbol{\varphi}_{t}^{T}\left(\sum_{i=1}^{n} \mathbf{M}_{i}^{\mathrm{ele}}\right) \boldsymbol{\varphi}_{t}},
\end{aligned}
$$

$$
\begin{aligned}
& \lambda_{i(3), t}=\frac{\boldsymbol{\varphi}_{t}^{T}\left(I_{y i} \mathbf{K}_{i(3)}^{\mathrm{ele}}\right) \boldsymbol{\varphi}_{t}}{\boldsymbol{\varphi}_{t}^{T}\left(\sum_{i=1}^{n} \mathbf{M}_{i}^{\mathrm{ele}}\right) \boldsymbol{\varphi}_{t}}, \\
& \lambda_{i(4), t}=\frac{\boldsymbol{\varphi}_{t}^{T}\left(J_{i} \mathbf{K}_{i(4)}^{\mathrm{ele}}\right) \boldsymbol{\varphi}_{t}}{\boldsymbol{\varphi}_{t}^{T}\left(\sum_{i=1}^{n} \mathbf{M}_{i}^{\mathrm{ele}}\right) \boldsymbol{\varphi}_{t}} .
\end{aligned}
$$

4.2. The Sensitivity of Frequency to Interval Parameters. For uncertain structures, some of the parameters in (16) are uncertain values. Now, assuming that the material parameters $E_{i} \in\left[E_{i}^{\text {low }}, E_{i}^{\text {up }}\right], \mu_{i} \in\left[\mu_{i}^{\text {low }}, \mu_{i}^{\text {up }}\right], \rho_{i} \in\left[\rho_{i}^{\text {low }}, \rho_{i}^{\text {up }}\right]$ are uncertain and treated as interval variables, where $E_{i}^{\text {low }}, \mu_{i}^{\text {low }}$, and $\rho_{i}^{\text {low }}$ are the lower bounds and $E_{i}^{\text {up }}, \mu_{i}^{\text {up }}$, and $\rho_{i}^{\text {up }}$ are the upper bounds of the interval parameters, respectively, then an investigation on the frequency sensitivity of material parameters is expanded.

Take the partial derivatives with respect to the interval variables $E_{i}, \mu_{i}$, and $\rho_{i}$ in (16). Considering that the error in the computed frequency by the Rayleigh quotient is proportional to the square of the deviation of the standard modes from their exact values [23], the effect of the modification of the standard mode on the natural frequency is a second-order small quantity. So that the effect can be ignored when taking the partial derivatives. Then the frequency sensitivities can be obtained:

$$
\begin{aligned}
& \frac{\partial \lambda_{t}}{\partial E_{i}}=\lambda_{i(1), t}+\lambda_{i(2), t}+\lambda_{i(3), t}+\frac{\lambda_{i(4), t}}{2\left(1+\mu_{i}\right)}, \\
& \frac{\partial \lambda_{t}}{\partial \mu_{i}}=-\frac{E_{i}}{2\left(1+\mu_{i}\right)^{2}} \lambda_{i(4), t},
\end{aligned}
$$




$$
\begin{aligned}
& \frac{\partial \lambda_{t}}{\partial \rho_{i}} \\
& \quad=-\frac{\boldsymbol{\varphi}_{t}^{T}\left(A_{i} \mathbf{M}_{i(1)}^{\mathrm{ele}}+A_{i} \mathbf{M}_{i(2)}^{\mathrm{ele}}+A_{i} \mathbf{M}_{(3)}^{\mathrm{ele}}+J_{i} \mathbf{M}_{i(4)}^{\mathrm{ele}}\right) \boldsymbol{\varphi}_{t}}{\boldsymbol{\varphi}_{t}^{T}\left(\sum_{i=1}^{n} \mathbf{M}_{i}^{\mathrm{ele}}\right) \boldsymbol{\varphi}_{t}} \\
& \quad \cdot \sum_{i=1}^{n}\left(E_{i} \lambda_{i(1), t}+E_{i} \lambda_{i(2), t}+E_{i} \lambda_{i(3), t}\right. \\
& \left.+\frac{E_{i}}{2\left(1+\mu_{i}\right)} \lambda_{i(4), t}\right) .
\end{aligned}
$$

4.3. Frequency Analysis Based on Monotonicity Discriminant of the Frequency Sensitivity. The interval ranges of the natural frequencies can be obtained if the frequency sensitivities are monotonic. The monotonicity analysis of the frequency sensitivities is carried out as follows.

Considering the situation that the geometric parameters are certain parameters, then $A_{i}, I_{z i}, I_{y i}, J_{i}$, and $L_{i}$ are all certain positive values. According to (16) and (18), the global mass matrix $\mathbf{M}$ is positive definite, and the component matrices $\mathbf{K}_{i(1)}^{\mathrm{ele}}, \mathbf{K}_{i(2)}^{\mathrm{ele}}, \mathbf{K}_{i(3)}^{\mathrm{ele}}, \mathbf{K}_{i(4)}^{\mathrm{ele}}, \mathbf{M}_{i(1)}^{\mathrm{ele}}, \mathbf{M}_{i(2)}^{\mathrm{ele}}, \mathbf{M}_{i(3)}^{\mathrm{ele}}$, and $\mathbf{M}_{i(4)}^{\mathrm{ele}}$ are given in (14) and (15). Based on the knowledge of linear algebra, a sufficient and necessary condition of positive semidefinite for the real symmetric matrices is that all of the principal minors are nonnegative. Checked by calculations, in (14), the nonzero principal minors of $\mathbf{K}_{i(1)}^{\text {ele }}, \mathbf{K}_{i(2)}^{\text {ele }}, \mathbf{K}_{i(3)}^{\text {ele }}$, and $\mathbf{K}_{i(4)}^{\text {ele }}$ are all nonnegative numbers; in (15), the nonzero principal minors of $\mathbf{M}_{i(1)}^{\text {ele }}, \mathbf{M}_{i(3)}^{\text {ele }}$, and $\mathbf{M}_{i(4)}^{\text {ele }}$ are all positive numbers and the nonzero principal minors of $\mathbf{M}_{i(2)}^{\text {ele }}$ are all nonnegative numbers; therefore, the component matrices $\mathbf{K}_{i(1)}^{\text {ele }}, \mathbf{K}_{i(2)}^{\text {ele }}, \mathbf{K}_{i(3)}^{\text {ele }}, \mathbf{K}_{i(4)}^{\text {ele }}, \mathbf{M}_{i(1)}^{\text {ele }}, \mathbf{M}_{i(2)}^{\text {ele }}, \mathbf{M}_{i(3)}^{\text {ele }}$, and $\mathbf{M}_{i(4)}^{\text {ele }}$ turn out to be positive semidefinite.

According to the above results of deducing, we can know that $\lambda_{i(1), t}, \lambda_{i(2), t}, \lambda_{i(3), t}$, and $\lambda_{i(4), t}$ in (16) are all nonnegative. After careful investigation towards (18), the values of the frequency sensitivity are obtained, where $\partial \lambda_{t} / \partial E_{i}$ is nonnegative and $\partial \lambda_{t} / \partial \mu_{i}$ and $\partial \lambda_{t} / \partial \rho_{i}$ are nonpositive.

As the monotonicity of frequency sensitivity is obtained, the maximums and minimums of the frequencies can be expressed as follows by utilizing the generalized Rayleigh quotient in (12):

$$
\begin{aligned}
& \lambda_{t \text { max }}=\frac{\boldsymbol{\varphi}_{t \max }^{T} \mathbf{K}\left(E_{i}^{\text {up }}, \mu_{i}^{\text {low }}\right) \boldsymbol{\varphi}_{t \max }}{\boldsymbol{\varphi}_{t \text { max }}^{T} \mathbf{M}\left(\rho_{i}^{\text {low }}\right) \boldsymbol{\varphi}_{t \max }}, \\
& \lambda_{t \text { min }}=\frac{\boldsymbol{\varphi}_{t \text { min }}^{T} \mathbf{K}\left(E_{i}^{\text {low }}, \mu_{i}^{\text {up }}\right) \boldsymbol{\varphi}_{t \text { min }}}{\boldsymbol{\varphi}_{t \text { min }}^{T} \mathbf{M}\left(\rho_{i}^{\text {up }}\right) \boldsymbol{\varphi}_{t \text { min }}},
\end{aligned}
$$

where $\varphi_{t \text { max }}$ and $\varphi_{t \text { min }}$ are the $t$ th order standard modes when $\lambda_{t}$ gets the maximum and minimum values, respectively. $\lambda_{t}$ gets the maximum value $\lambda_{t \max }$ when $\rho_{i}, \mu_{i}$ get the lower bounds $\rho_{i}^{\text {low }}, \mu_{i}^{\text {low }}$ and simultaneously $E_{i}$ gets the upper bound $E_{i}^{\mathrm{up}} ; \lambda_{t}$ gets the minimum value $\lambda_{t \text { min }}$ when $\rho_{i}, \mu_{i}$ get the upper bounds $\rho_{i}^{\text {up }}, \mu_{i}^{\text {up }}$ and simultaneously $E_{i}$ gets the lower bound $E_{i}^{\text {low }}$.

4.4. Optimization Algorithm to Calculate the Frequency Interval. For uncertain structure with bounded parameters, the square of the $t$ th order natural frequency $\lambda_{t}$ in (12) is in a certain interval range. Then the purpose of frequency analysis is to identify the interval ranges of the natural frequencies. Generally, this interval problem could be solved by an optimization algorithm. The optimization model can be established as follows:

$$
\begin{aligned}
\min \lambda_{t} & =\frac{\boldsymbol{\varphi}_{t}^{T} \mathbf{K}(\mathbf{X}) \boldsymbol{\varphi}_{t}}{\boldsymbol{\varphi}_{t}^{T} \mathbf{M}(\mathbf{X}) \boldsymbol{\varphi}_{t}}, \\
\max \quad \lambda_{t} & =\frac{\boldsymbol{\varphi}_{t}^{T} \mathbf{K}(\mathbf{X}) \boldsymbol{\varphi}_{t}}{\boldsymbol{\varphi}_{t}^{T} \mathbf{M}(\mathbf{X}) \boldsymbol{\varphi}_{t}} \\
\text { s.t. } \quad \mathbf{X} & =\left\{X_{j}\right\}, \quad X_{j} \in\left[X_{j}^{L}, X_{j}^{U}\right], j=1,2, \ldots, m,
\end{aligned}
$$

where $X_{j}^{L}$ denotes the lower bound of $X_{j}$ and $X_{j}^{U}$ denotes the upper bound of it. The global stiffness matrix $\mathbf{K}(\mathbf{X})$ and global mass matrix $\mathbf{M}(\mathbf{X})$ are functions of the interval variables vector $\mathbf{X}$. By solving the optimization problems, the upper and lower bounds of the natural frequencies can be obtained.

Theoretically, an approximate value of the exact solution could be obtained via solving the former optimization model in (20). The optimization model is applicable to various uncertain structures, so it is utilized here to verify the correctness of the former presented monotonicity discriminant method.

Particle Swarm Optimization (PSO) is a metaheuristic evolutionary optimization technique which can be directly applied in a continuous global space environment and was first proposed by Kennedy and Eberhart [24]. PSO is quite popular in the swarm intelligence community due to its simplest algorithmic structure, less parameter use, and being free from gradient use of an objective function. The PSO algorithm starts with randomly initialized population. It works on the social behavior of particle to get the best solution by adjusting each individual's position which can respect the global best position of the whole population. Each individual is adjusting by changing the velocity according to its own experience and by observing the position of the other particles by use of (21) [25]. Consider

$$
\begin{aligned}
v_{i}(k+1)=\omega & \cdot v_{i}(k)+a_{1} \cdot r_{1} \cdot\left(p_{i \text { best }}-x_{i}(k)\right)+a_{2} \\
& \cdot r_{2} \cdot\left(g_{\text {best }}-x_{i}(k)\right), \\
x_{i}(k+1)= & x_{i}(k)+v_{i}(k+1), \\
& \quad i=1,2, \ldots, N ; k=1,2, \ldots, I-1,
\end{aligned}
$$

where $\omega$ is the inertia weight in $[0.4,0.9] ; a_{1}$ and $a_{2}$ are the acceleration factors in [0,2] and $r_{1}$ and $r_{2}$ are random numbers in $[0,1] ; N$ is the population size and $I$ is the stated iteration times; $v_{i}(k)$ denotes the velocity of the $i$ th particle after the $k$ th iteration and $x_{i}(k)$ denotes the position of the $i$ th particle after the $k$ th iteration; $p_{i \text { best }}$ is the best position 


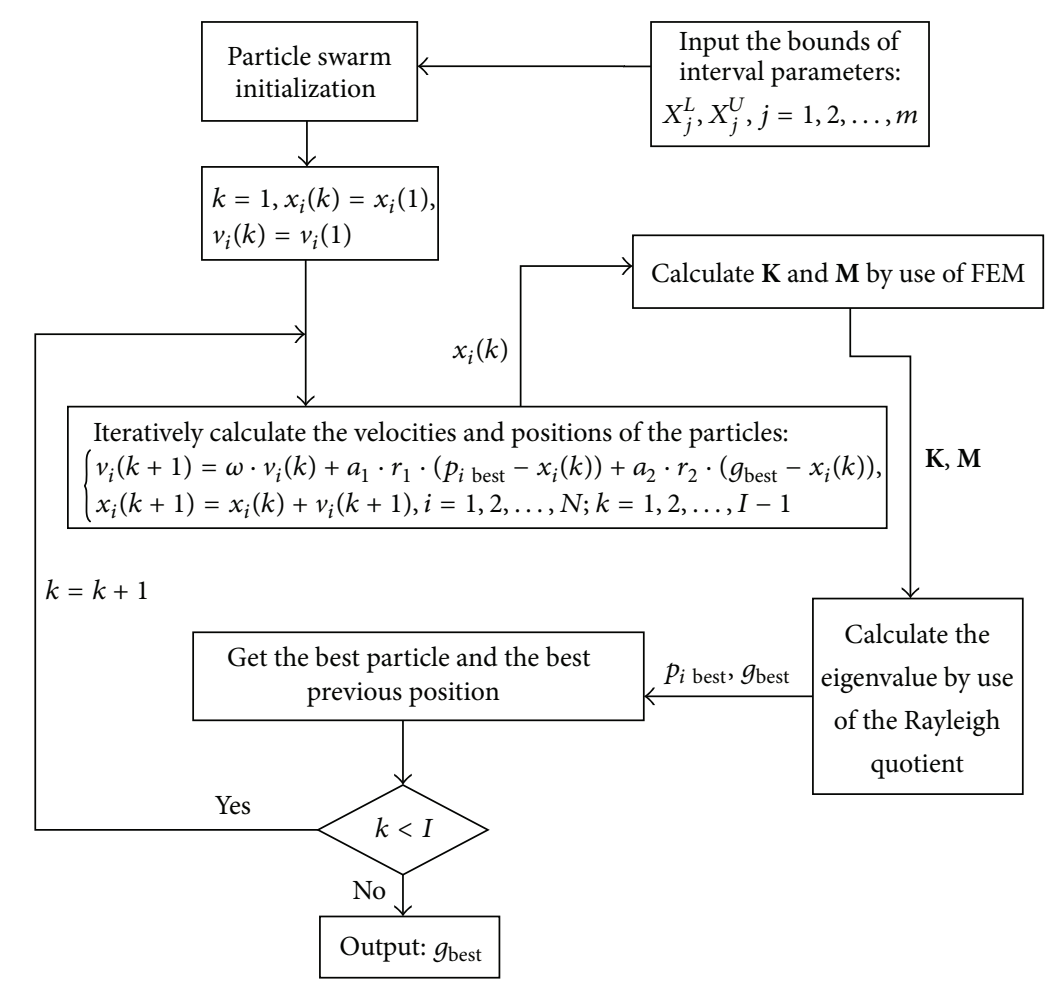

FIGURE 5: Flowchart of the PSO procedure to calculate ranges of the natural frequencies.

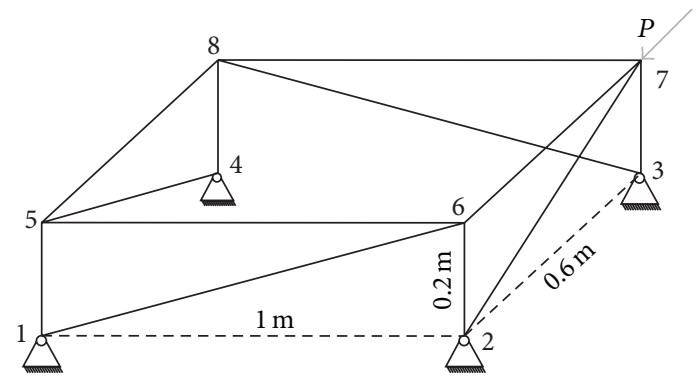

FIGURE 6: 12-element space frame structure.

of the $i$ th particle and $g_{\text {best }}$ is the global best position in the population.

In this paper, a standard PSO algorithm is applied to calculate the maximums and minimums of the natural frequencies. The flowchart of the procedure for realization of the optimization process of (20) is depicted in Figure 5. After iteration calculation, the extremums of the natural frequencies can be worked out. It also can be seen that $N \times I$ times of finite element analysis need to be accomplished, which may reduce the efficiency of calculation.

\section{Examples}

5.1. A Space Frame Structure. The space frame structure (shown in Figure 6) is considered in this example. The frame is composed of 12 space beams. The sectional area of each beam is $0.01 \times 0.01 \mathrm{~m}^{2}$. The elasticity modulus $E$, Poisson's
TABLE 1: Natural intervals of the space frame structure.

\begin{tabular}{lcc}
\hline Frequencies & $\begin{array}{c}\text { Sensitivity monotonicity } \\
\text { method }(\mathrm{Hz})\end{array}$ & $\begin{array}{c}\text { Optimization } \\
\text { algorithm }(\mathrm{Hz})\end{array}$ \\
\hline$\left[\omega_{1}^{L}, \omega_{1}^{U}\right]$ & {$[51.0809,54.3731]$} & {$[51.0809,54.3731]$} \\
{$\left[\omega_{2}^{L}, \omega_{2}^{U}\right]$} & {$[64.1601,68.2445]$} & {$[64.1601,68.2445]$} \\
{$\left[\omega_{3}^{L}, \omega_{3}^{U}\right]$} & {$[75.9927,80.7316]$} & {$[75.9927,80.7316]$} \\
{$\left[\omega_{4}^{L}, \omega_{4}^{U}\right]$} & {$[78.8702,83.8342]$} & {$[78.8702,83.8342]$} \\
{$\left[\omega_{5}^{L}, \omega_{5}^{U}\right]$} & {$[94.4190,100.4346]$} & {$[94.4190,100.4346]$} \\
{$\left[\omega_{6}^{L}, \omega_{6}^{U}\right]$} & {$[124.0793,131.9354]$} & {$[124.0793,131.9354]$} \\
\hline
\end{tabular}

ratio $\mu$, and density $\rho$ are interval variables, where $E \in$ $[200,220] \mathrm{Gpa}, \mu \in[0.29,0.31]$, and $\rho \in[7760,7960] \mathrm{Kg} / \mathrm{m}^{3}$. The node 7 is under transverse load with the frequency $P$ in an interval range $[58,62] \mathrm{Hz}$.

Respectively, process the frequency analysis on the frame structure by use of the sensitivity monotonicity algorithm and the PSO. The structure was divided into 12 beam elements with 8 nodes. Then the natural frequency intervals were worked out. The first 6 order intervals are shown in Table 1. According to the sensitivity monotonicity method in Section 4 , the natural frequencies get the maximum values when the elasticity modulus $E$ is on the upper bound meanwhile the Poisson ratio $\mu$ and density $\rho$ are on the lower bounds, and the natural frequencies get the minimum values when the elasticity modulus $E$ is on the lower bound meanwhile the Poisson ratio $\mu$ and density $\rho$ are on the upper bounds. From Table 1, we can find that the sensitivity monotonicity method and the optimization algorithm have 


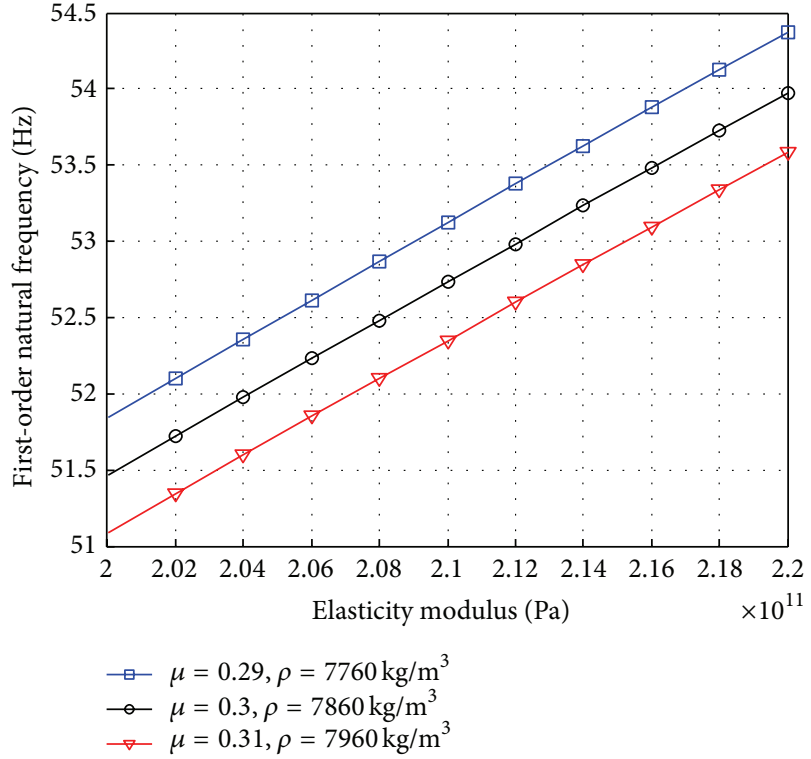

(a)

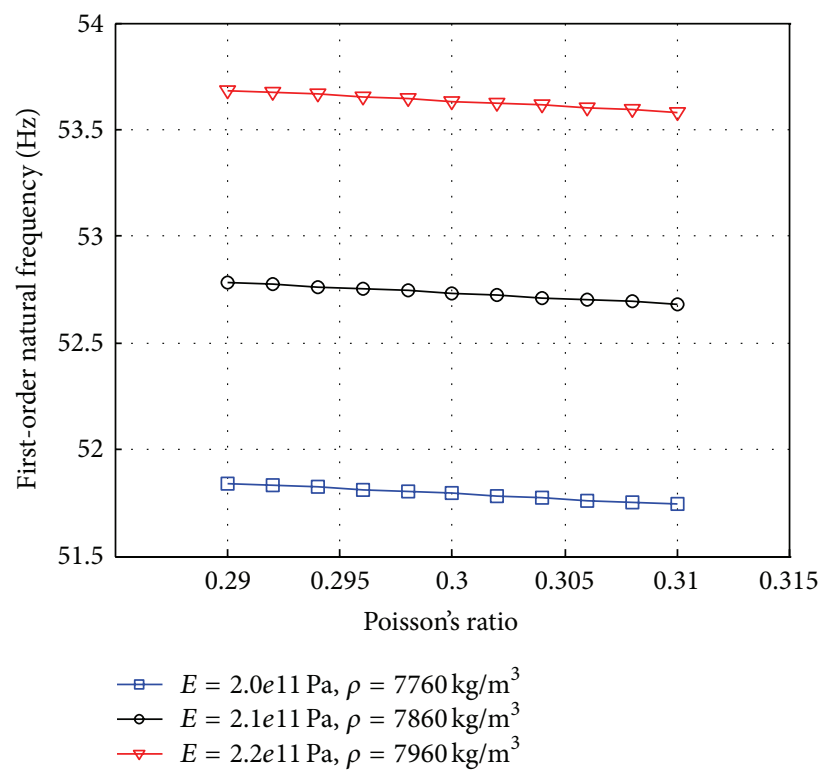

(b)

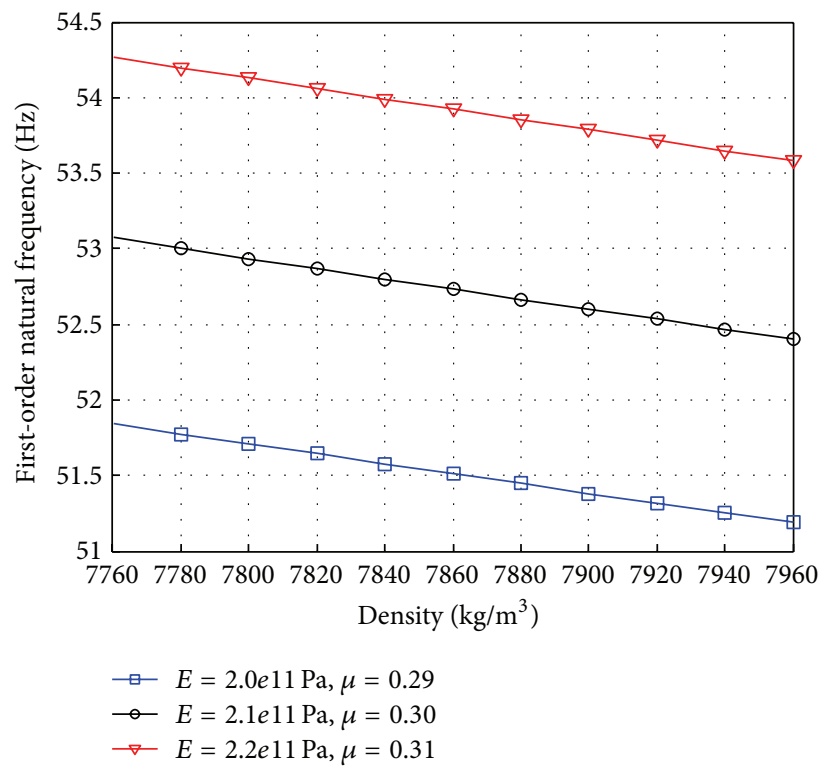

(c)

FIGURE 7: The change of the first-order natural frequency along with the variables of the frame. (a) The change along with elasticity modulus. (b) The change along with Poisson's ratio. (c) The change along with density.

the same results. Considering that the PSO has nice global searching capability, the result of the PSO is close to the exact solution. So the validity of the sensitivity monotonicity method presented in this paper is verified.

In addition, the sensitivity monotonicity method just experienced two times of FEM analysis to obtain the result, but the optimization algorithm in Section 4.4 took $20 \times 5$ times of FEM analysis away (20 represents the population; 5 represents the evolution times). So the sensitivity monotonicity method has much higher efficiency.

The changes of the first-order natural frequency along with the interval variables of the frame structure are shown in Figure 7. It can be seen that the frequency increases with the increasing of elasticity modulus and decreases with the increasing of Poisson's ratio and density, which shows the validity of the monotonicity of frequency sensitivity.

Already known are the minimal distance $\gamma=1.5 \mathrm{~Hz}$, excitation frequency $P \in[58,62] \mathrm{Hz}$, and the range of natural frequencies shown in Table 1 . Transform the frequencies into standard ones. Then the system reliability index $\eta_{\text {sys }}$ can be calculated out according to (9), (10), and (11). The value of the system reliability index is 1.1633 . The index is the distance from the origin to the lower boundary of the failure domain which is formed by $P$ and $\omega_{2}$. The value of the index indicates 


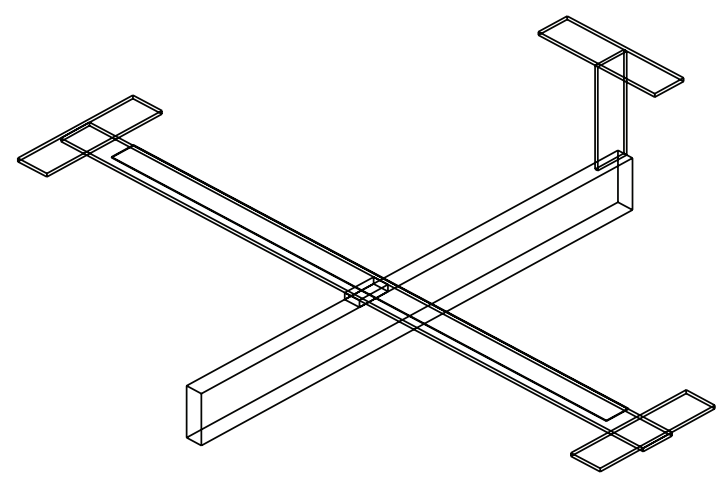

(a)

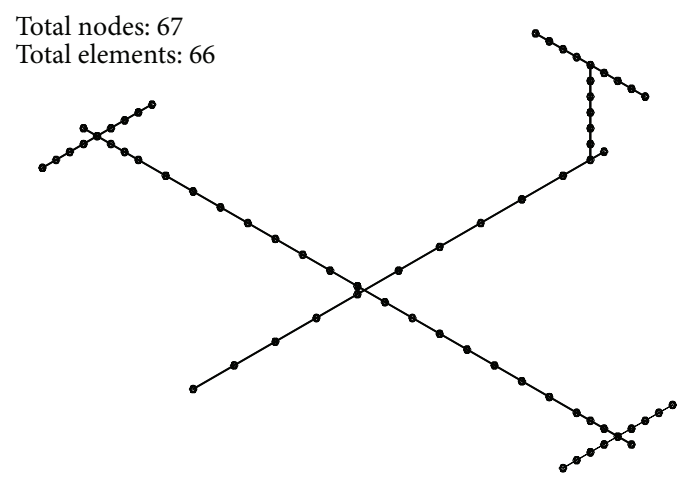

(b)

FIgURE 8: A simplified GARTEUR plane model. (a) GARTEUR plane model. (b) Finite element model of the GARTEUR plane.

TABLE 2: Natural frequency intervals of GARTEUR model.

\begin{tabular}{lcc}
\hline Frequencies & $\begin{array}{c}\text { Sensitivity monotonicity } \\
\text { method }(\mathrm{Hz})\end{array}$ & $\begin{array}{c}\text { Optimization } \\
\text { algorithm }(\mathrm{Hz})\end{array}$ \\
\hline$\left[\omega_{1}^{L}, \omega_{1}^{U}\right]$ & {$[5.1252,5.2395]$} & {$[5.1252,5.2393]$} \\
{$\left[\omega_{2}^{L}, \omega_{2}^{U}\right]$} & {$[5.1954,5.2452]$} & {$[5.1955,5.245]$} \\
{$\left[\omega_{3}^{L}, \omega_{3}^{U}\right]$} & {$[24.2211,24.2211]$} & {$[24.2211,24.2211]$} \\
{$\left[\omega_{4}^{L}, \omega_{4}^{U}\right]$} & {$[33.4799,35.495]$} & {$[33.4799,35.4919]$} \\
{$\left[\omega_{5}^{L}, \omega_{5}^{U}\right]$} & {$[38.8969,39.3312]$} & {$[38.8971,39.3310]$} \\
{$\left[\omega_{6}^{L}, \omega_{6}^{U}\right]$} & {$[41.1152,42.6744]$} & {$[41.1160,42.6744]$} \\
\hline
\end{tabular}

that the space frame structure is avoiding resonance while the excitation frequency $P$ is loading on the structure.

5.2. The GARTEUR Plane Model. GARTEUR plane model was developed by the French Aerospace Research Institute in 1995. The model has the same characteristic with the actual aircraft such as high flexibilities and modal density [26]. As shown in Figure 8(a), the model has a total length of $1.5 \mathrm{~m}$ and a wingspan of $2.0 \mathrm{~m}$, the material is aluminum, and there is a damping layer attached to the wing. The density, elastic modulus, and Poisson ratio of the wing changed due to the existence of damping layer [27]. The whole model is simplified to a space beam model, and there are a total of 67 nodes and 66 beam elements; the restricted model is shown in Figure 8(b). The elastic modulus of the connection element was set as interval variable $E_{c} \in[100,1000] \mathrm{GPa}$ after examining the simplification of the connection between the wing and the fuselage. Considering the existence of the damping layer, the elastic modulus of the wing was set as interval variable $E_{w} \in[67.5,68.5] \mathrm{GPa}$, the density of the wing as interval variable $\rho_{w} \in[2700,2900] \mathrm{Kg} / \mathrm{m}^{3}$, and Poisson's ratio of the wing as interval variable $\mu_{w} \in[0.29,0.31]$. The gust frequency loaded on the wing is assumed as $P \in[10,15] \mathrm{Hz}$.

By use of the sensitivity monotonicity method and the optimization algorithm, the frequency analysis was, respectively, carried out on the GARTEUR plane model. The frequency intervals were, respectively, obtained by calculating. The first 6 order intervals are shown in Table 2. From Table 2 we can find that the results of the two methods are nearly the same. Considering that the result of the PSO is close to the exact solution, the validity of the sensitivity monotonicity method is verified.

In addition, the sensitivity monotonicity method only experienced two times of FEM analysis to obtain the results, but the optimization algorithm in Section 4.4 took $20 \times 10$ times of FEM analysis away (20 represents the population; 10 represents the evolution times). So the sensitivity monotonicity method is much more efficient.

The changes of the first-order natural frequency along with the interval variables of the GARTEUR plane model are shown in Figure 9. It can be seen that the frequency increases with the increasing of elasticity modulus of the wing and decreases with the increasing of density of the wing. The elasticity modulus of the connection and Poisson's ratio of the wing have little impact on the first-order natural frequency. It is caused by the special structure of the connection and the wing. The analysis result of Figure 9 also shows the validity of the monotonicity of frequency sensitivity.

Already known are the minimal distance $\gamma=1.5 \mathrm{~Hz}$, the gust frequency $P \in[8,14] \mathrm{Hz}$, and the range of natural frequencies shown in Table 2 . Transform the frequencies into standard ones. Then the system reliability index $\eta_{\text {sys }}$ can be calculated out according to (9), (10), and (11). The value of the system reliability index is 1.4123 . The index is the distance from the origin to the upper boundary of the failure domain which is formed by $P$ and $\omega_{1}$. This value of the index indicates that the GARTEUR plane model is avoiding resonance while the gust frequency is loading on the wing.

\section{Conclusion}

Considering the facts that available information about the uncertain parameters is often limited in practical engineering and it is relatively easy to construct a conservative interval model, a series system vibration reliability based on the interval nonprobabilistic reliability is introduced to avoid resonance of the structures with uncertain-but-bounded parameters in this paper. According to the interval theory, the failure domains similar to infinite strips are presented and the LSSs are obtained corresponding to the different relationship between the natural frequencies and the excitation 


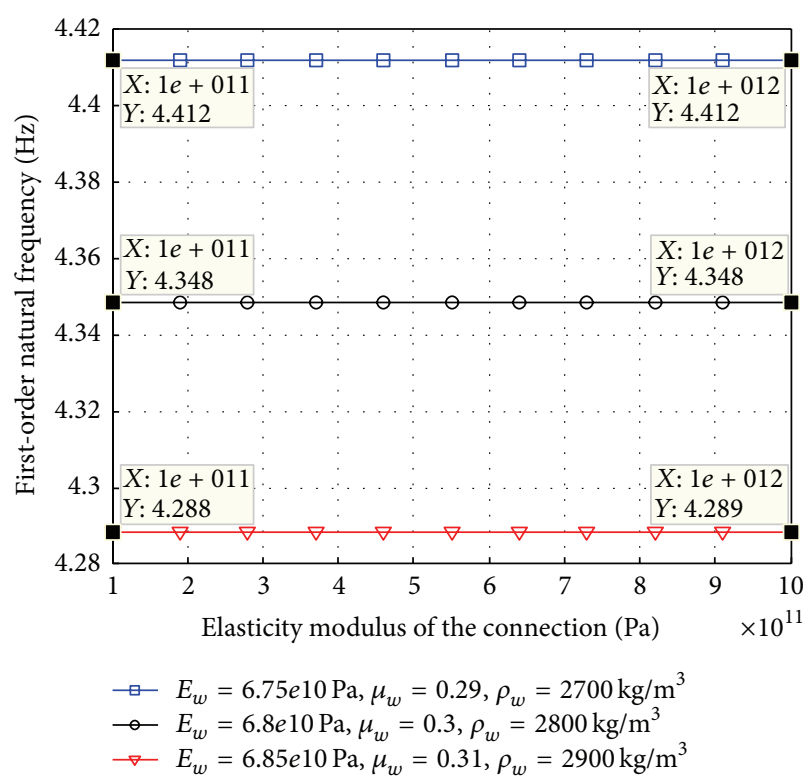

(a)

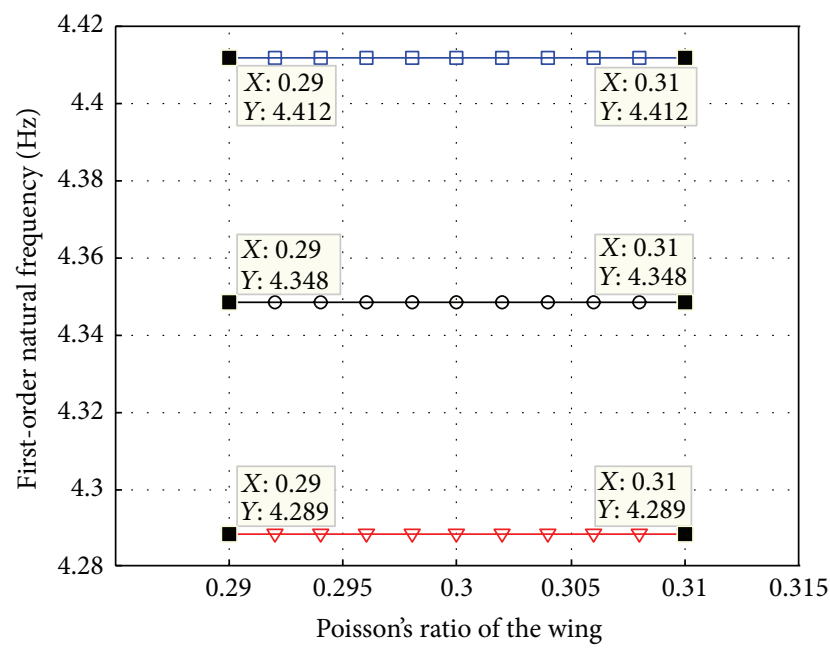

$\square E_{c}=1 e 11 \mathrm{~Pa}, E_{w}=6.75 e 10 \mathrm{~Pa}, \rho_{w}=2700 \mathrm{~kg} / \mathrm{m}^{3}$
$\rightarrow-E_{c}=5.5 e 11 \mathrm{~Pa}, E_{w}=6.8 e 10 \mathrm{~Pa}, \rho_{w}=2800 \mathrm{~kg} / \mathrm{m}^{3}$
$\rightarrow-E_{c}=1 e 12 \mathrm{~Pa}, E_{w}=6.85 e 10 \mathrm{~Pa}, \rho_{w}=2900 \mathrm{~kg} / \mathrm{m}^{3}$

(c)

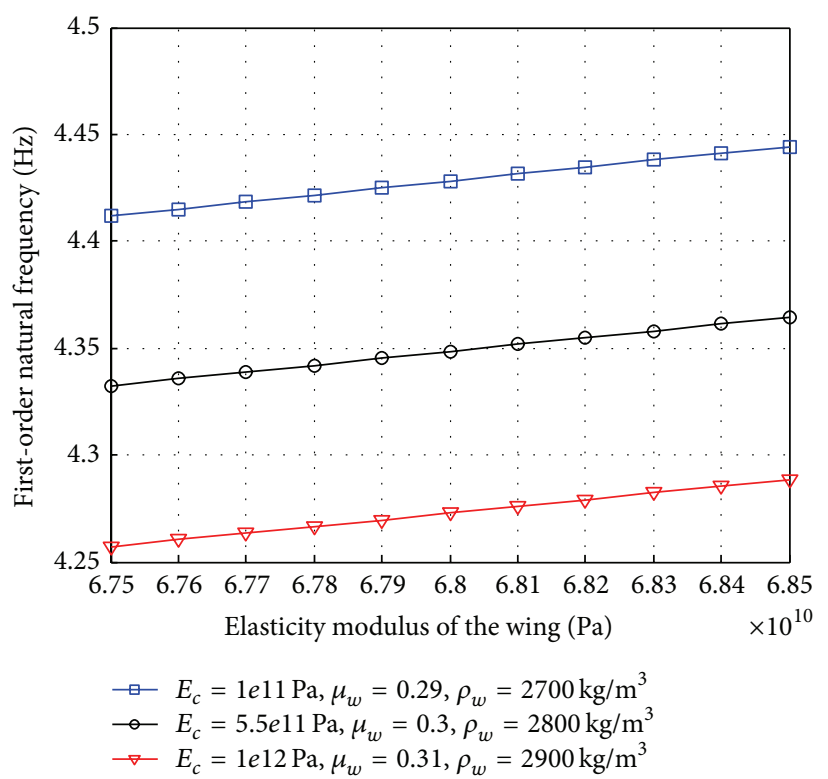

(b)

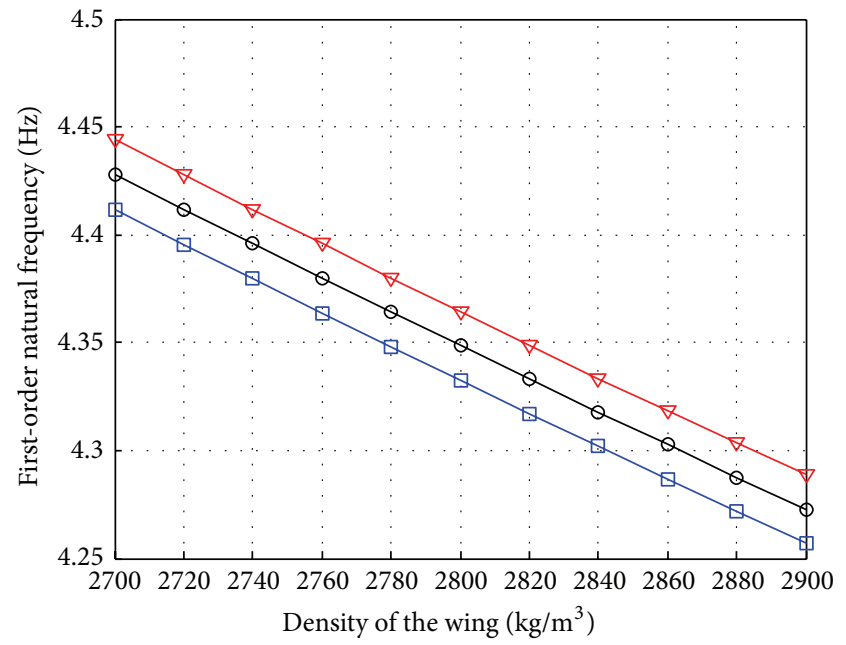

$\rightarrow E_{c}=1 e 11 \mathrm{~Pa}, E_{w}=6.75 e 10 \mathrm{~Pa}, \mu_{w}=0.29$
$\rightarrow-E_{c}=5.5 e 11 \mathrm{~Pa}, E_{w}=6.8 e 10 \mathrm{~Pa}, \mu_{w}=0.30$
$\rightarrow E_{c}=1 e 12 \mathrm{~Pa}, E_{w}=6.85 e 10 \mathrm{~Pa}, \mu_{w}=0.31$

(d)

FIGURE 9: The change of the first-order natural frequency along with the variables of the GARTEUR model. (a) The change along with elasticity modulus of the connection. (b) The change along with elasticity modulus of the wing. (c) The change along with Poisson's ratio of the wing. (d) The change along with density of the wing.

frequencies. Therefore, the series system vibration reliability index is obtained. Although this series system has the same failure mechanism with the traditional stochastic series system, it has a different system reliability index due to the different variable types. The series system vibration reliability index represents the shortest distance from the origin to the LSSs which are the boundaries of the failure domain. It also can be regarded as a robust reliability index because the structure is much more robust when the index is larger. In order to get the failure modes of the uncertain structure, a frequency analysis is needed. In this paper, the space beam structure with material parameters as the interval variables is considered as the object. A frequency analysis method based on the monotonicity discriminant of the frequency sensitivity is presented. This method only needs two times of FEM analysis to calculate the upper and lower bounds of the frequencies, so it is much more efficient. Then the global optimization algorithm PSO is introduced to verify the validity of the method. At last, a space frame structure and a simplified GARTEUR plane model are provided to illustrate the validity and feasibility of the presented method. 


\section{Conflict of Interests}

The authors declare that there is no conflict of interests regarding the publication of this paper.

\section{Acknowledgment}

The support from the National Natural Science Foundation of China (Grant no. 51175510) is gratefully acknowledged.

\section{References}

[1] I. Elishakoff, "Essay on uncertainties in elastic and viscoelastic structures: from A. M. Freudenthal's criticisms to modern convex modeling," Computers and Structures, vol. 56, no. 6, pp. 871-895, 1995.

[2] Y. Ben-Haim and I. Elishakoff, Convex Models of Uncertainty in Applied Mechanics, Elsevier Science Publisher, Amsterdam, The Netherlands, 1990.

[3] G. Shuxiang, L. Zhenzhou, and F. Yuansheng, "A non-probabilistic model of structural reliability based on interval analysis," Chinese Journal of Computational Mechanics, vol. 18, no. 1, pp. 56-60, 2001 (Chinese).

[4] I. Elishakoff and Y. J. Ren, Finite Element Methods for Structures with Large Stochastic Variations, chapter 7, Oxford University Press, Oxford, UK, 2003.

[5] J.-B. Yang and H.-L. Sun, "Discrete method for structural interval reliability analysis," in Proceedings of the Chinese Control and Decision Conference (CCDC '08), pp. 2441-2446, Yantai, China, July 2008.

[6] W. Sun, R. Dong, and H. Xu, "A novel non-probabilistic approach using interval analysis for robust design optimization," Journal of Mechanical Science and Technology, vol. 23, no. 12, pp. 3199-3208, 2009.

[7] C. Jiang, G. Y. Lu, X. Han, and L. X. Liu, "A new reliability analysis method for uncertain structures with random and interval variables," International Journal of Mechanics and Materials in Design, vol. 8, no. 2, pp. 169-182, 2012.

[8] C. Jiang, Q. F. Zhang, X. Han, and Y. H. Qian, "A non-probabilistic structural reliability analysis method based on a multidimensional parallelepiped convex model," Acta Mechanica, vol. 225, no. 2, pp. 383-395, 2014.

[9] S.-X. Guo and Y. Li, "Non-probabilistic reliability method and reliability-based optimal LQR design for vibration control of structures with uncertain-but-bounded parameters," Acta Mechanica Sinica, vol. 29, no. 6, pp. 864-874, 2013.

[10] S.-X. Guo and Z.-Z. Lu, "A non-probabilistic robust reliability method for analysis and design optimization of structures with uncertain-but-bounded parameters," Applied Mathematical Modelling, vol. 39, no. 7, pp. 1985-2002, 2015.

[11] Y. Zhang, Q. Liu, and B. Wen, "Quasi-failure analysis on resonant demolition of random structural systems," AIAA Journal, vol. 40, no. 3, pp. 585-586, 2002.

[12] C. Su, Y. Zhang, and Q. Zhao, "Vibration reliability sensitivity analysis of general system with correlation failure modes," Journal of Mechanical Science and Technology, vol. 25, no. 12, pp. 3123-3133, 2011.

[13] J. Rohn, "Eigenvalues of a symmetric interval matrix," Freiburger Intervall-Berichte, vol. 87, pp. 67-72, 1987.
[14] A. S. Deif, Advanced Matrix Theory for Scientists and Engineers, Abacus Press, Tunbridge Wells, UK, 1991.

[15] Z.-P. Qiu, S.-H. Chen, and J.-X. Na, “The Rayleigh quotient method for computing eigenvalue bounds of vibrational systems with interval parameters," Acta Mechanica Solida Sinica, vol. 6, no. 3, pp. 309-318, 1993.

[16] Z. Qiu, I. Elishakoff, and J. H. Starnes Jr., "The bound set of possible eigenvalues of structures with uncertain but nonrandom parameters," Chaos, Solitons \& Fractals, vol. 7, no. 11, pp. 1845-1857, 1996.

[17] I. Elishakoff, Ed., Whys and Hows in Uncertainty Modelling: Probability, Fuzziness and Anti-Optimization, vol. 388 of CISM Courses and Lectures, Springer, Vienna, Austria, 1999.

[18] W. Gao, "Interval natural frequency and mode shape analysis for truss structures with interval parameters," Finite Elements in Analysis and Design, vol. 42, no. 6, pp. 471-477, 2006.

[19] M. Modares, R. L. Mullen, and R. L. Muhanna, "Natural frequencies of a structure with bounded uncertainty," Journal of Engineering Mechanics, vol. 132, no. 12, pp. 1363-1371, 2006.

[20] A. Sofi, G. Muscolino, and I. Elishakoff, "Natural frequencies of structures with interval parameters," Journal of Sound and Vibration, vol. 347, pp. 79-95, 2015.

[21] Y. Zhang, B. Wen, and Q. Liu, "First passage of uncertain single degree-of-freedom nonlinear oscillators," Computer Methods in Applied Mechanics and Engineering, vol. 165, no. 1-4, pp. 223231, 1998.

[22] L. Witek, "Simulation of crack growth in the compressor blade subjected to resonant vibration using hybrid method," Engineering Failure Analysis, vol. 49, pp. 57-66, 2015.

[23] W. T. Thomson and M. D. Dahleh, Theory of Vibration with Application, chapter 12, section 1, Prentice-Hall, Englewood Cliffs, NJ, USA, 5th edition, 1998.

[24] J. Kennedy and R. C. Eberhart, "Particle swarm optimization," in Proceedings of the 4th IEEE International Conference on Neural Networks, pp. 1942-1948, December 1995.

[25] H. Das, A. K. Jena, J. Nayak, B. Naik, and H. S. Behera, "A novel PSO based back propagation learning-MLP (PSO-BP-MLP) for classification," in Computational Intelligence in Data MiningVolume 2, vol. 32, pp. 461-471, Springer, 2015.

[26] H. De Gersem, D. Moens, W. Desmet, and D. Vandepitte, "Interval and fuzzy dynamic analysis of finite element models with superelements," Computers \& Structures, vol. 85, no. 5-6, pp. 304-319, 2007.

[27] M. Degener and M. Hermes, "Ground vibration test and finite element analysis of the GARTEUR SM-AG19 testbed," DLR Report IB 232-96 J08, 1996. 


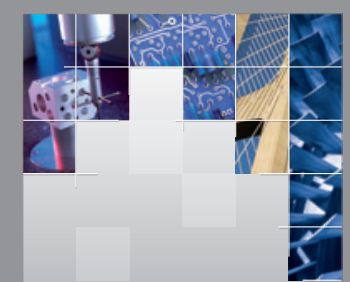

\section{Enfincering}
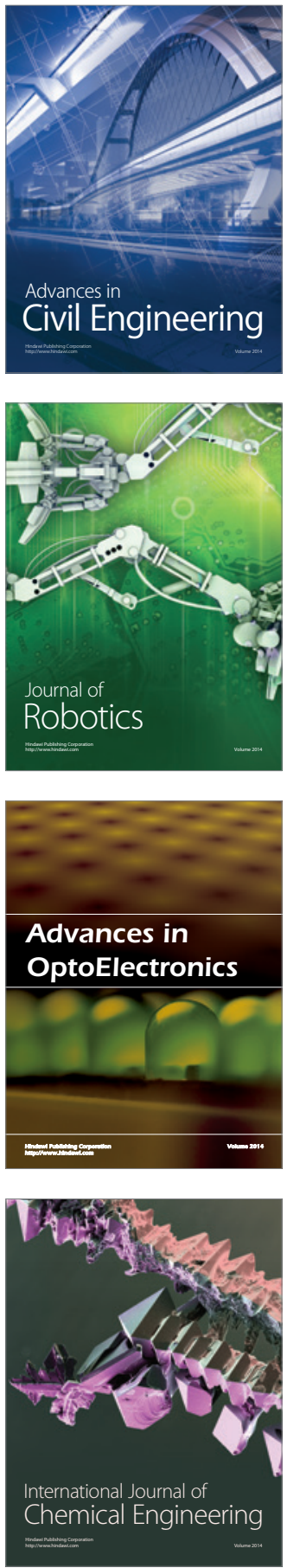

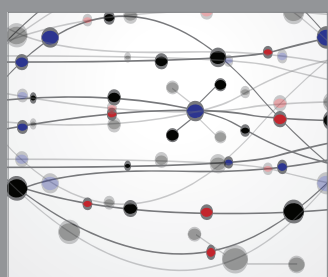

The Scientific World Journal

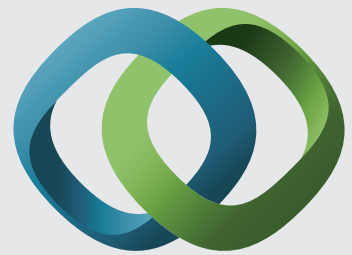

\section{Hindawi}

Submit your manuscripts at

http://www.hindawi.com
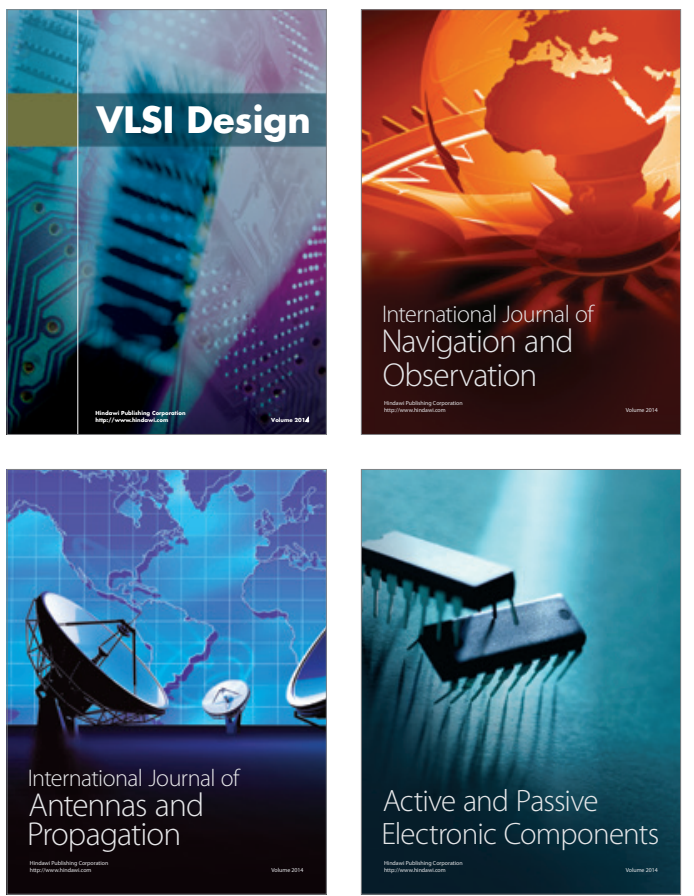
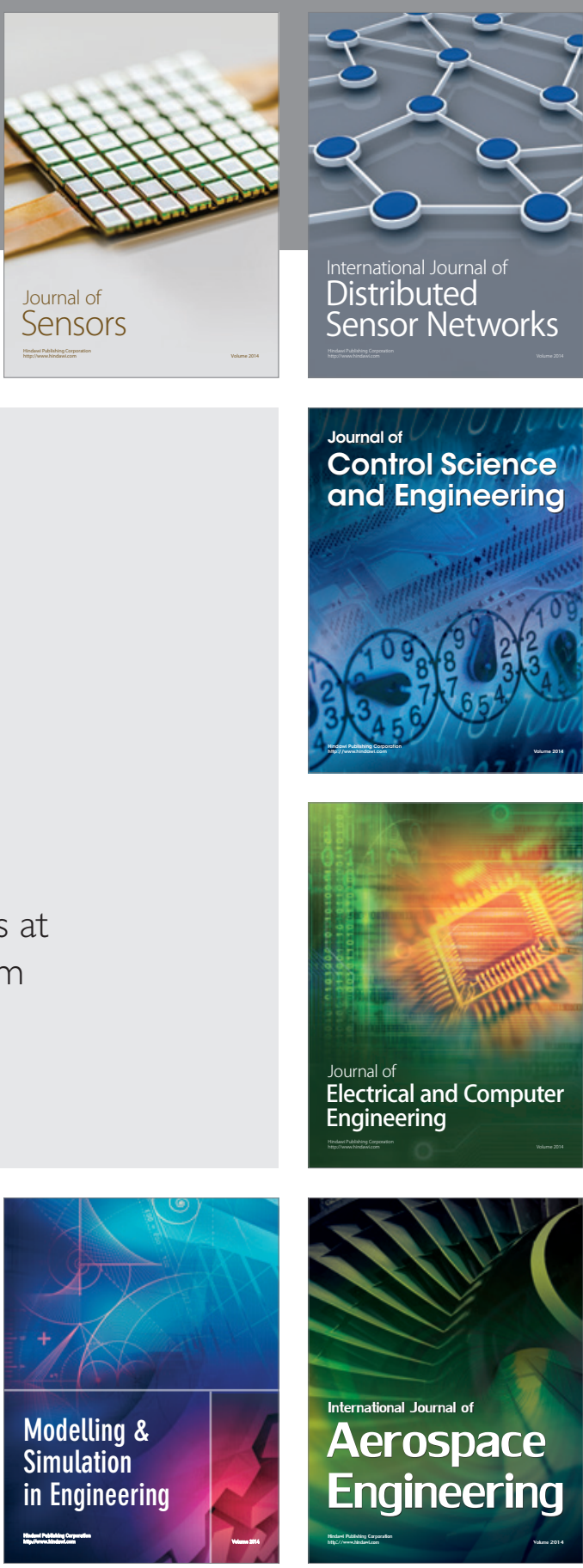

International Journal of

Distributed

Sensor Networks

Journal of

Control Science

and Engineering
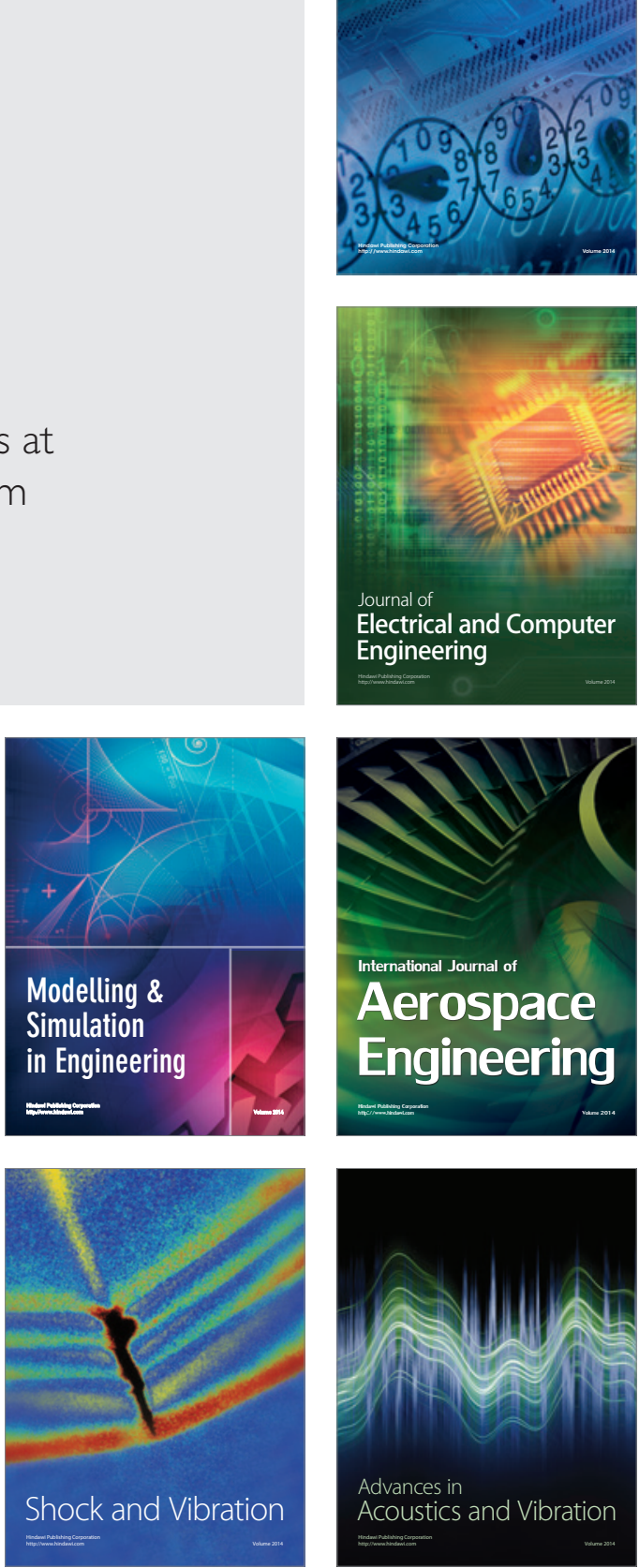